\title{
EHMT1 regulates Parvalbumin-positive interneuron development and GABAergic input in sensory cortical areas
}

\author{
Moritz Negwer $^{1} \cdot$ Karol Piera $^{1} \cdot$ Rick Hesen $^{1} \cdot$ Lukas Lütje $^{1} \cdot$ Lynn Aarts $^{1} \cdot$ Dirk Schubert $^{2} \cdot$ Nael Nadif Kasri $^{1,2}$ (i)
}

Received: 12 February 2020 / Accepted: 10 September 2020 / Published online: 25 September 2020

(c) The Author(s) 2020

\begin{abstract}
Mutations in the Euchromatic Histone Methyltransferase 1 (EHMT1) gene cause Kleefstra syndrome, a rare form of intellectual disability (ID) with strong autistic traits and sensory processing deficits. Proper development of inhibitory interneurons is crucial for sensory function. Here we report a timeline of Parvalbumin-positive $\left(\mathrm{PV}^{+}\right)$interneuron development in the three most important sensory cortical areas in the $E h m t 1^{+/-}$mouse. We find a hitherto unreported delay of $\mathrm{PV}^{+}$neuron maturation early in sensory development, with layer- and region-specific variability later in development. The delayed $\mathrm{PV}^{+}$maturation is also reflected in a delayed maturation of GABAergic transmission in $E h m t 1^{+/-}$auditory cortex, where we find a reduced GABA release probability specifically in putative $\mathrm{PV}^{+}$synapses. Together with earlier reports of excitatory impairments in Ehmt ${ }^{+/-}$neurons, we propose a shift in excitatory-inhibitory balance towards overexcitability in $E h m t 1^{+/-}$sensory cortices as a consequence of early deficits in inhibitory maturation.
\end{abstract}

Keywords Parvalbumin-positive interneurons $\cdot$ Kleefstra syndrome $\cdot$ Gabaergic synapse $\cdot$ Critical period $\cdot$ Cortical development $\cdot$ Autism spectrum disorder $\cdot$ EHMT1

\section{Introduction}

Kleefstra Syndrome (KS, OMIM\#610253) is a rare syndromic form of intellectual disability (ID) associated with autistic features (Kleefstra et al. 2005, 2012; Koemans et al. 2017; Vermeulen et al. 2017), caused by haploinsufficiency of the EHMT1 gene. The EHMT1 protein (also known as GLP or KMT1D) functions as a repressive epigenetic modifier by mono- and dimethylating euchromatic Histone 3 on Lysine 9 (H3K9me1/2; Benevento et al. 2015; Iwase et al. 2017; Shinkai and Tachibana 2011; Tachibana et al. 2005). Mice with a heterozygous loss-of-function mutation

Electronic supplementary material The online version of this article (https://doi.org/10.1007/s00429-020-02149-9) contains supplementary material, which is available to authorized users.

Nael Nadif Kasri

n.nadif@donders.ru.nl

1 Department of Human Genetics, Radboudumc, Donders Institute for Brain, Cognition, and Behaviour, 6500 HB Nijmegen, The Netherlands

2 Department of Cognitive Neuroscience, Radboudumc, Donders Institute for Brain, Cognition and Behaviour, 6500 HB Nijmegen, The Netherlands in the Ehmtl gene recapitulate the core phenotypes of KS, including low muscle tone, delayed development of sensory modalities and craniofacial abnormalities (Balemans et al. 2010, 2014). Ehmtl ${ }^{+-}$mice show a delayed onset of sensory experiences (eye opening) and sensory reaction (acoustic startle response; Balemans et al. 2014), as well as impaired learning and exploration, reduced locomotor activity, and increased anxiety (Balemans et al. 2013). This phenotype is partially replicated in excitatory-neuron specific Ehmt 1 knockout mice (CaMKIIa-Cre), which also present with hypoactivity and learning deficits, but do not show an anxiety-like phenotype (Schaefer et al. 2009).

On a physiological level, we previously reported hyperexcitability in Ehmt $1^{+/}$hippocampal excitatory neurons (Frega et al. 2020) as well as deficits in evoked excitatory transmission in auditory cortex (Frega et al. 2019). Those excitatory phenotypes are broadly replicated in cultured neurons derived from both rodents and human KS patientderived iPS cells (Frega et al. 2019; Martens et al. 2016). Ehmtl $^{+/-}$neurons show a deficit in homeostatic synaptic scaling (upscaling at excitatory synapses) in response to input deprivation, both in primary cultures and in the visual cortex (Benevento et al. 2016). On a molecular level, Ehmt 1 and its homolog Ehmt2 co-regulate activity-dependent gene 
expression in the hippocampus and amygdala (Gupta-Agarwal et al. 2012, 2014).

Interestingly, the deficit in sensory development observed in Ehmt1 ${ }^{+-}$mice (Balemans et al. 2014) was not observed in excitatory-neuron specific Ehmtl conditional knockout mice (Ehmt $f^{f l o x /+} \times$ CaMKIIa-Cre; Schaefer et al. 2009), suggesting an inhibitory component for the developmental deficit. This hypothesis is strengthened by recent physiological evidence of an inhibitory impairment in the Ehmt $^{+/-}$hippocampal stratum oriens (Frega et al. 2020), which is enriched in $\mathrm{PV}^{+}$basket cells (Booker and Vida 2018), indicating $\mathrm{PV}^{+}$neurons as a possible target subpopulation. In sensory cortical areas, $\mathrm{PV}^{+}$interneurons set the pace for the maturation of the entire circuit, which might explain the sensory developmental deficit observed in $E h \mathrm{mtl}^{+/-}$mice. However, the influence of Ehmtl on the trajectory of different inhibitory interneuron classes remains unknown.

Parvalbumin-positive $\left(\mathrm{PV}^{+}\right)$interneurons are the largest group of GABAergic interneurons (releasing $\gamma$-amino butyric acid) in the cortex and directly control firing in their target cells (Tremblay et al. 2016). They mature relatively late during cortical development, during a time of heightened sensitivity to environmental influences known as the critical period (Hensch 2005; Marín 2016; Reh et al. 2020; Trachtenberg 2015). During the critical period, $\mathrm{PV}^{+}$neurons start expressing the calcium-buffering protein PV and generate perineuronal nets (PNNs), a honeycomb-like extracellular matrix structure that elevates their firing rate and effectively locks in their excitatory input at this timepoint (Favuzzi et al. 2019; Sigal et al. 2019; Testa et al. 2019). The timing of $\mathrm{PV}^{+}$interneuron maturation is plastic and depends on sensory input (Marín 2016). Interestingly, mouse models for ID and autism spectrum disorders (ASD) show temporal shifts in both directions: earlier maturation in $M e c p 2^{-/ y}$ mice, a mouse model for Rett Syndrome (Krishnan et al. 2015, 2017; Patrizi et al. 2019), and delayed maturation in $\mathrm{Fmrl}^{-/ y}$ mice, a mouse model for Fragile X Syndrome (Goel et al. 2018; Harlow et al. 2010; Reinhard et al. 2019). Strikingly, $\mathrm{PV}^{+}$neuron-specific knockout of Mecp 2 prevented a critical period altogether (Banerjee et al. 2016; He et al. 2014), underlining $\mathrm{PV}^{+}$neuron development in sensory cortices as a possible point of convergence in neurodevelopmental disorders.

Here, we investigated the developmental trajectory of $\mathrm{PV}^{+}$neurons in the three largest primary sensory cortical areas (A1, S1, V1), at three relevant timepoints for their respective development. In order to quantify inhibitory neurotransmission, we also measured the output of $\mathrm{PV}^{+}$neurons onto pyramidal neurons in auditory cortex layer $2 / 3$. We find that in $\mathrm{Ehmt1}^{+/-}$mice, $\mathrm{PV}^{+}$neuron maturation is delayed at postnatal day (P) 14 in layer 4 in all regions examined. With the exception of $\mathrm{S} 1$ layer $4, \mathrm{PV}^{+}$maturation markers in
$E h m t^{+/-}$mice normalize to wild-type levels later in development. Genetic labelling of $\mathrm{PV}^{+}$cells showed no difference in any region in adulthood, indicating a dynamic effect of Ehmtl on $\mathrm{PV}^{+}$expression. In agreement with this delay of $\mathrm{PV}^{+}$neuron maturation, we find a reduction of inhibitory synaptic function in the Ehmt1 ${ }^{+/-}$auditory cortex at P14. As a cause, we identify a reduced release probability for GABAergic synapses, specifically from fast-spiking (putative $\mathrm{PV}^{+}$) neurons.

\section{Materials and methods}

\section{Animals}

For the animal experiments presented in this study, mice heterozygous for a targeted loss-of-function mutation in the Ehmtl gene (Ehmt1 ${ }^{+/-}$mice; Tachibana et al. 2005) and their wild-type littermates $\left(\mathrm{Ehmtl}^{+/+}\right)$on a C57BL/6 background were used, as previously described (Balemans et al. 2010; Benevento et al. 2016). We used mice from both sexes for our study. Animals were kept in wire-top cages (type III), group-housed with either the mother animals with her nest until weaning (P21-25), and afterwards group-housed (3-6 animals, littermates, both genotypes, segregated by sex) in wire-top type III cages. Animals had access to rodent chow and water ad libitum and were kept on a $12 \mathrm{~h} / 12 \mathrm{~h}$ light-/ dark cycle (lights on at 07:00 a.m.). Animal experiments were conducted in conformity with the Animal Care Committee of the Radboud University Nijmegen Medical Centre and the National Committee on Animal Experiments (CCD), The Netherlands, and conform to the guidelines of the Dutch Council for Animal Care and the European Communities Council Directive 2010/63/EU.

\section{Immunostaining}

Ehmt $^{+/+}$and ${ }^{+/-}$littermates of both sexes were harvested in one of three age groups: P14, P28, and P56. Animals were deeply anesthetized with isoflurane, then decapitated and the brain was quickly extracted. The brains were fixed overnight in $4 \%$ PFA with $4 \%$ Sucrose in PBS at $4{ }^{\circ} \mathrm{C}$ and kept in PBS at $4{ }^{\circ} \mathrm{C}$ until slicing. For slicing, the brains were embedded in $1.5 \%$ Agarose in PBS, and $60 \mu \mathrm{m}$ coronal sections were cut on a vibratome (Leica VT1000s). The slices were matched to the Allen Adult Mouse Brain Atlas (Atlas version 2, Oh et al. 2014; https://atlas.brain-map.org/atlas?atlas $=1$ ) and kept in PBS with $0.001 \%$ Sodium Azide at $4{ }^{\circ} \mathrm{C}$.

For expression of PV and PNNs, $60 \mu \mathrm{m}$ free-floating slices were stained overnight at room temperature with Rabbit anti-PV (Swant 27, 1:500) and biotinylated Wisteria Floribunda Agglutinin (WFA, Sigma L1516, 1:500), followed by Donkey anti-Rabbit Alexa 488 (Invitrogen 
A21206, 1:500), and Streptavidin-Alexa 568 (Molecular Probes S11226, 1:500) for $3 \mathrm{~h}$ at room temperature, and counterstained with Hoechst 33,342 (1:5000, 15 min).

For synapse staining P14 slices containing primary auditory cortex were stained as described earlier (van Rhijn et al. 2018), including an antigen retrieval step with $0.05 \%$ Trypsin in PBS ( $37{ }^{\circ} \mathrm{C}$ for $\left.15 \mathrm{~min}\right)$. Slices were then incubated for $72 \mathrm{~h}$ at $4{ }^{\circ} \mathrm{C}$ with Chicken Anti-NeuN (Millipore ABN91, 1:750) and Mouse Anti-Gad67 (Merck Millipore MAB 5406, 1:500), followed by $3 \mathrm{~h}$ at room temperature with Goat anti-chicken 568 (Invitrogen A11041, 1:500), and Goat anti-mouse 647 (Invitrogen A21236, 1:500). Nuclei were counterstained with Hoechst 33,342 (1:5000, 15 min).

\section{Fluorescence microscopy}

The PV/PNN stained slices were imaged at $5 \times$ and $40 \times$ on a Zeiss AxioImager.Z1 with Apotome $(40 \times$ only). We used Zeiss EC Plan-Neofluar $5 \times / 0.16$ M27 and Zeiss EC Plan-Neofluar 40x/0.75 M27 objectives, and imaged with a Zeiss Axiocam 506. From each sensory cortex, we collected between 1 and 10 non-overlapping images (number matched within each region to account for differences in the size of the brain region) for both layer $2 / 3$ and 4 , from each slice. Per animal, we used 4 slices per area, and per timepoint $N=3-6$ each $E h m t 1^{+/+} / E h m t 1^{+/-}$mice, for a total of between 13 and 68 images (matched within one brain region) per genotype for each area and layer.

The NeuN/Gad65 stained slices were imaged at $5 \times$ and $63 \times$ on a Zeiss AxioImager.Z1 with Apotome unit (63×only). We used Zeiss EC Plan-Neofluar 5x/0.16 M27 and Zeiss Plan-Apochromat $63 \times / 1.4$ Oil DIC M27 objectives, and imaged with a Zeiss Axiocam 506. We used one timepoint (P14) with $N=3 / 3$ Ehmt $^{+/+} /$Ehmt $^{+/-}$mice, 4 slices per animal, and collected between 2 and 3 non-overlapping images of each auditory cortex layer $2 / 3$ at $63 \times$.

\section{Image analysis}

For the PV/PNN stainings, we processed the images with Zen (Blue edition, version 2.3, Zeiss) to export TIFF images. We used a custom-made ImageJ/FIJI script to randomize the file names, for blinding to the genotype. The images were analyzed semi-automatically with the PIPSQUEAK toolbox which is specifically written for PV/PNN overlap analysis (Slaker et al. 2016). Within PIPSQUEAK, we assigned the positions of $\mathrm{PV}^{+}$and $\mathrm{PNN}^{+}$neurons by hand without thresholding, and used PIPSQUEAK to assess PV/PNN overlap. PIPSQUEAK assignment was done blinded to the genotype. Un-blinding and further processing of the data was done in Excel, and statistics were done in Excel and Graphpad Prism (version 7). The overview images used in Figs. 1, 2 and $3 \mathrm{~b}$ were stitched from $5 \times$ Images with a custom-written
ImageJ macro using the ImageJ stitching plugin (Preibisch et al. 2009). For the NeuN/Gad65 stainings, we processed the images with Zen (Blue edition, version 2.3, Zeiss) to export TIFF images and counted perisomatic $\mathrm{Gad} 67^{+}$puncta with the CellCounter plugin of ImageJ/FIJI (Schindelin et al. 2012), and the resulting counts were processed in Excel.

\section{Whole-brain immunostaining, clearing and analysis}

For this protocol we crossed mice expressing the tdTomatoflox/flox conditional marker (B6.Cg-Gt(ROSA)26Sortm9(CAGtdTomato)Hze/J or Ai9; a kind gift from Michael Valente, RIMLS Nijmegen) with Ehmtl ${ }^{+/-}$mice until we had Ehmt $^{+/-}$; tdTomato ${ }^{\text {flox/flox }}$ males (Supplementary Fig. S2). Those were then crossed with $\mathrm{PV}$-Cre homozygote females (a kind gift from Tansu Celikel, Donders Institute Nijmegen) and the resulting litter-matched offspring $\left(\mathrm{PV}^{\mathrm{Crel} /} ;\right.$ tdTomato $^{\text {flox/- }}$ and either Ehmt ${ }^{+/+}$or Ehmt $\left.{ }^{ \pm}\right)$ were used for experiments. Those mice express tdTomato in cells once the $P V$ promoter has been active, and importantly, will keep expressing it even if $P V$ is downregulated again, allowing us to genetically target the superset of all potential $\mathrm{PV}^{+}$neurons in the cortex. At P56, the mice were sacrificed and brains were extracted and fixed as described above. We processed one hemisphere per brain following the iDISCO+ protocol (Renier et al. 2016) as described for adult brains, with all buffers according to the protocol and all incubation steps taking place on a shaker/rotor, and lasting $1 \mathrm{~h}$ unless mentioned otherwise. Briefly, the whole hemispheres dehydrated in a Methanol gradient, delipidated with 66\% Dichloromethane (DCM, Sigma)/33\% Methanol (Sigma) overnight, bleached in $5 \% \mathrm{H}_{2} \mathrm{O}_{2}$ in Methanol at $4 \mathrm{C}$ overnight, then rehydrated. Hemispheres were subsequently permeabilized for 5 days at RT, blocked for 4 days at $37^{\circ} \mathrm{C}$, then incubated with primary antibodies (Rabbit Anti-RFP, Rockland 600-401-379, 1:2000, $2 \mathrm{ml} / \mathrm{sample}$ ) for 6 days at $37^{\circ} \mathrm{C}$. Subsequently, brains were washed $5 \times 1 \mathrm{~h}+1 \times$ overnight at RT, and incubated for 7 days with secondaries (Goat anti-rabbit Alexa-568, Invitrogen A11036, 1:500, 2 ml/sample) at $37{ }^{\circ} \mathrm{C}$. Following $5 \times 1 \mathrm{~h}+1 \times$ overnight washing at RT, samples were dehydrated in a Methanol gradient, then twice more in $100 \%$ Methanol, $66 \%$ DCM/33\% Methanol, $2 \times 15 \mathrm{~min} 100 \% \mathrm{DCM}$, and finally cleared in $100 \%$ dibenzyl ether (DBE, Sigma) in airtight glass vials. Brains were typically transparent within $2 \mathrm{~h}$, and completely cleared overnight. Alexa-568 fluorescence remained undiminished until $>12$ months after clearing.

The cleared samples were imaged on a LaVision Ultramicroscope II Light-sheet microscope outfitted with a NTK Photonics white-light laser and filter sets for $488 \mathrm{~nm}$ and $568 \mathrm{~nm}$, imaged through a long-working distance objective (LaVision) at $1.1 \times$ magnification (effective 2.2x, NA 0.1), and recorded with an Andor Neo 5.5 
a

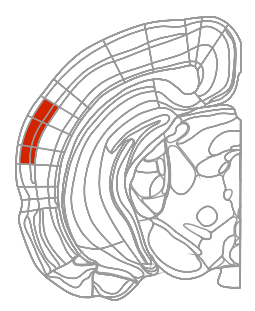

b

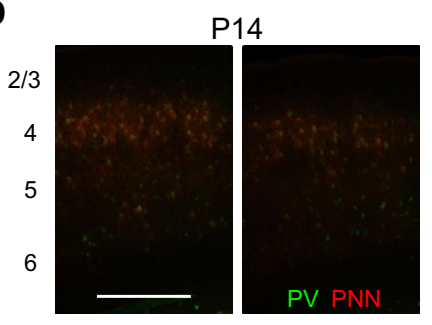

Ehmt1 $^{\text {+/+ }}$

Ehmt $1^{\text {+1 }}$

e Auditory Cortex, Layer 4

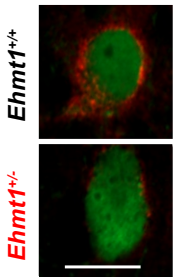

P14

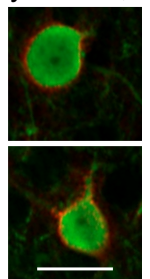

$\mathrm{P} 28$

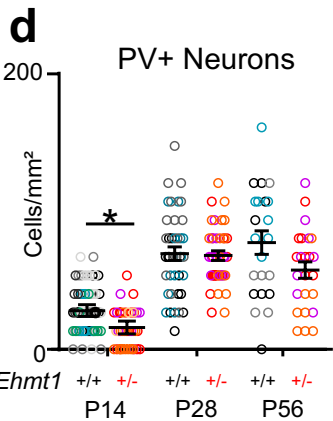

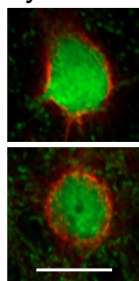

P56

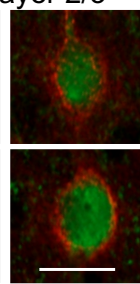

P56

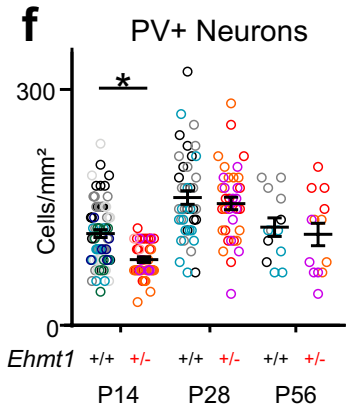

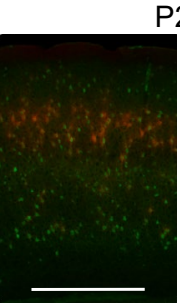

$\mathrm{Ehmt1}^{\text {+/+ }}$
P28

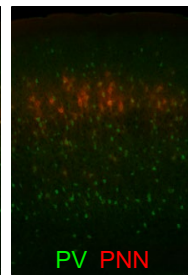

Ehmt1 ${ }^{+/-}$

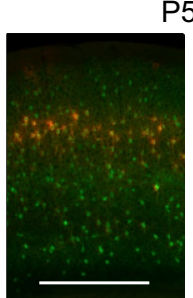

$E$ mt1 $^{+/+}$
56

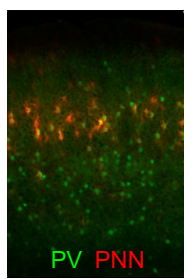

Ehmt1+/- $^{+1}$

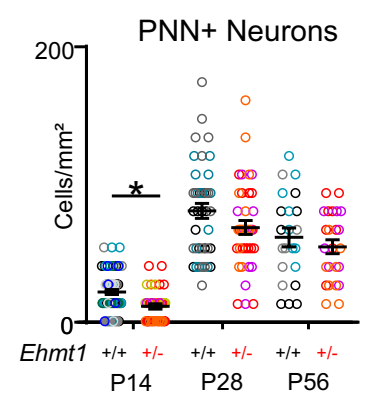

PNN+ Neurons

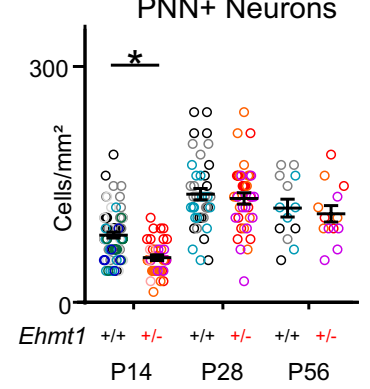

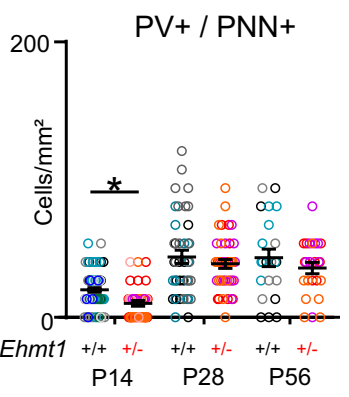

$\mathrm{PV}+/ \mathrm{PNN}+$

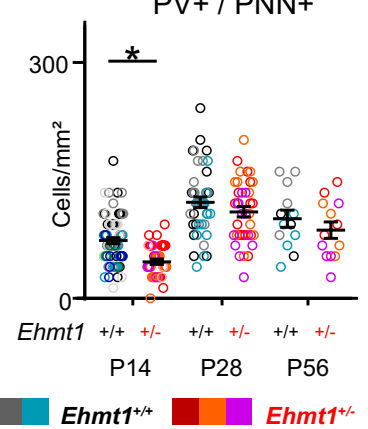

Fig. 1 Ehmt1 haploinsufficiency delays PV and PNN expression in the auditory cortex during the auditory critical period. a Primary auditory cortex, layers $2 / 3$ and 4 are marked in red. b Overview of $\mathrm{PV}^{+}$(green) and $\mathrm{PNN}^{+}$(red) development in the auditory cortex over time. Layer numbers are marked on the left. c Neurons stained for Parvalbumin (green) and PNNs (red) in the primary auditory cortex, layer 2/3. d Quantification of the density of $\mathrm{PV}^{+}, \mathrm{PNN}^{+}$, and $\mathrm{PV} /$ PNN double-labelled cells. N P14 $=63 / 38$, P28 $=40 / 39$, P56 $=22 / 24$ images, from 1 to 4 slices per animal, P14 $=6 / 4, \mathrm{P} 28=3 / 3, \mathrm{P} 56=3 / 3$ Ehmt $^{+/+} /$Ehmt $^{+/-}$animals. e Neurons stained for Parvalbumin (green) and PNNs (red) in the primary auditory cortex, layer $4 . \mathbf{f}$

cooled sCMOS camera. We imaged with a $480 \mathrm{~nm}$ signal for autofluorescence for alignment with the Allen Brain Atlas, and $560 \mathrm{~nm}$ to record the PV-TdTomato signal. We used a single light-sheet from one side at 0.54 NA, scanning at 2.95/2.95/3 $\mu \mathrm{m} x / y / z$ resolution ( $3 \mu \mathrm{m} z$-steps) with the "horizontal focus" method and 17-18 horizontal focus steps. The sample was imaged submerged in DBE in sagittal configuration, and the entire cortex fit inside a single field of view $(x / y)$, with a typical brain producing $\sim 1600$ $z$-planes of $3 \mu \mathrm{m}$ each. The resulting image stack was
Quantification of the density of $\mathrm{PV}^{+}, \mathrm{PNN}^{+}$, and $\mathrm{PV} / \mathrm{PNN}$ doublelabelled cells. N P14 $=66 / 38$, P28 $=40 / 37$, P56 $=13 / 13$ images, from 1 to 4 slices per animal, P14=6/4, P28 =3/3, P56=3/3 Ehmt ${ }^{+/+}$/ Ehmt1 ${ }^{+/-}$animals. Source for a Allen Adult Mouse Brain Atlas, https ://atlas.brain-map.org/atlas?atlas=1. Image Credit: Allen Institute. Scale bars in b $500 \mu \mathrm{m}, \mathbf{c}, \mathbf{e} 20 \mu \mathrm{m}$. Note that the intensities in c, e are matched within a timepoint, but not across timepoints. Scale in d, f cells $/ \mathrm{mm}^{2}$, Dot plot with mean \pm SEM, color-coded per mouse. ${ }^{*} p<0.05, * * p<0.01$; Nested ANOVA with mice as subgroups. See Supplementary Table 1 for exact values

de-striped in ImageJ, and fed into a heavily customized version of ClearMap 1 (Renier et al. 2016), with the cells being identified in Arivis Vision4D (Arivis $\mathrm{GmbH}$, https ://www.arivis.com/) using the "Machine Learning Segmenter" plugin, and cell locations being re-imported to ClearMap for alignment to the atlas and cell counts per region. We analyzed specifically the primary Auditory, Somatosensory, and Visual cortices, as displayed in Supplementary Figure S2. Visualizations were created in Arivis Vision4D and ImageJ, graphs and statistics were done with Excel. 
a

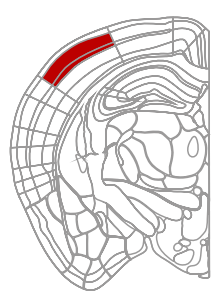

b

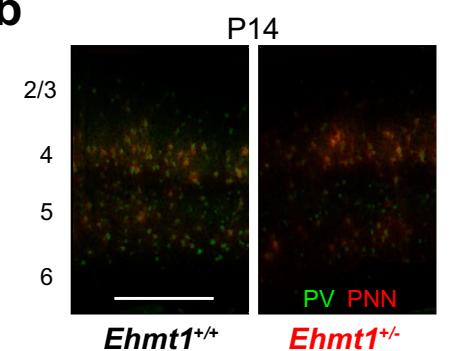

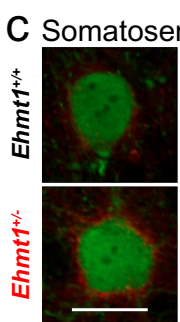

P14

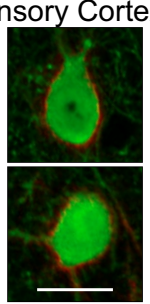

$\mathrm{P} 28$

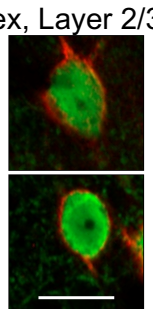

P56

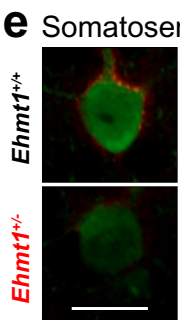

P14

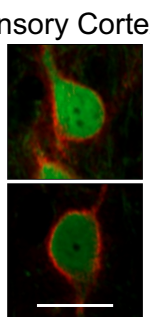

P28

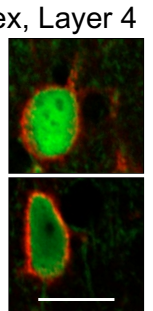

P56

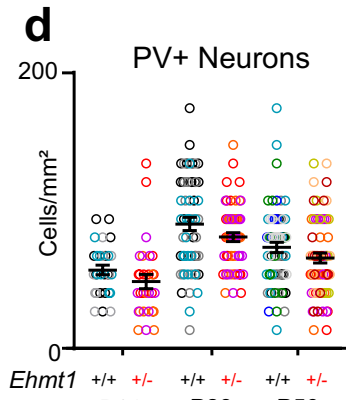

P14 P28 P56

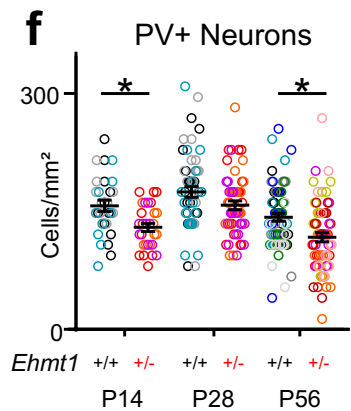

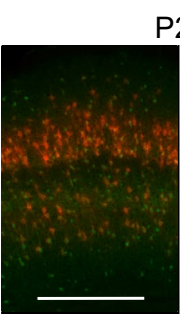

Ehmt1 $^{+++}$

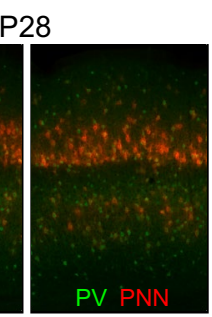

Ehmt1 $^{+/-}$

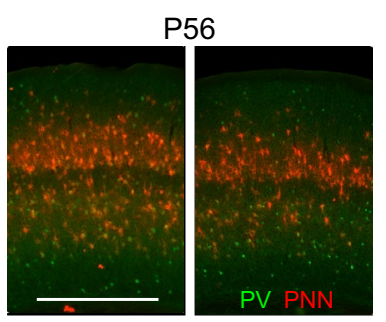

Ehmt1 $^{\text {t+ }}$

Ehmt1+/- $^{+1}$
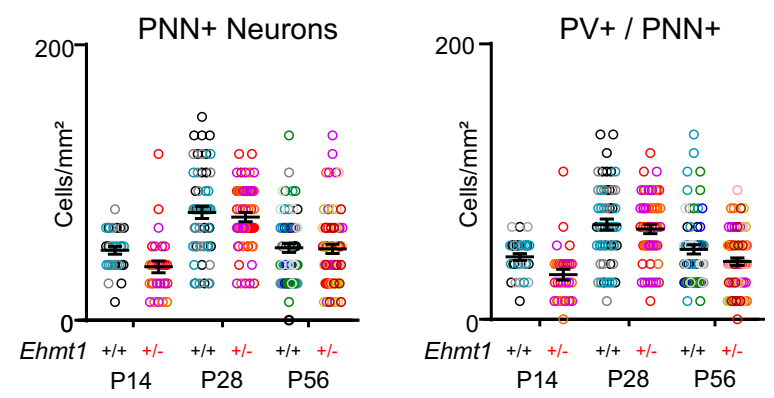

PNN+ Neurons

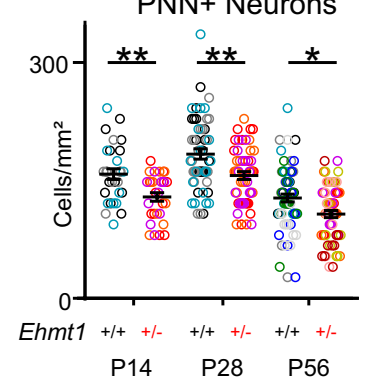

Fig. 2 A persistent reduction in $\mathrm{PV}^{+}$neuron markers in Ehmt ${ }^{+/-}$ somatosensory cortex layer 4 , but not in layer $2 / 3$. a Primary somatosensory cortex, Barrel field, layers $2 / 3$ and 4 are marked in red. b Overview of $\mathrm{PV}^{+}$(green) and $\mathrm{PNN}^{+}$(red) development in the somatosensory cortex over time. Layer numbers are marked on the left. c Neurons stained for Parvalbumin (green) and PNNs (red) in the primary somatosensory cortex, layer 2/3. d Quantification of the density of $\mathrm{PV}^{+}, \mathrm{PNN}^{+}$, and PV/PNN double-labelled cells. N P14=30/28, $\mathrm{P} 28=54 / 53, \mathrm{P} 56=63 / 63$ images, four slices each from $\mathrm{P} 14=3 / 3$, $\mathrm{P} 28=3 / 3, \mathrm{P} 56=6 / 6$ Ehmt $^{+/+} /$Ehmt $^{+/-}$animals. Color-code per mouse. e: Neurons stained for Parvalbumin (green) and PNNs (red)

\section{Acute slice electrophysiology}

Acute slice electrophysiology was performed as described earlier (Frega et al. 2019). We used litter-matched Ehmt ${ }^{+/+}$ and $E h m t 1^{+/-}$mice of adolescent age (postnatal day 14-16). In brief, animals were deeply anesthetized with isoflurane, then quickly decapitated. $350-\mu \mathrm{m}$-thick coronal slices were cut using a microtome (HM650V, Thermo Scientific) in icecold "cutting and storage" ACSF containing $87 \mathrm{mM} \mathrm{NaCl}$, $11 \mathrm{mM}$ Glucose, $75 \mathrm{mM}$ Sucrose, $2.5 \mathrm{mM} \mathrm{KCl}, 1.25 \mathrm{mM}$ $\mathrm{NaH}_{2} \mathrm{PO}_{4}, 0.5 \mathrm{mM} \mathrm{CaCl}_{2}, 7 \mathrm{mM} \mathrm{MgCl}$, and $26 \mathrm{mM}$ $\mathrm{NaHCO}_{3}$, continuously oxygenated with $95 \% \mathrm{O}_{2} / 5 \% \mathrm{CO}_{2}$. in the primary somatosensory cortex, layer 4. f Quantification of the density of $\mathrm{PV}^{+}, \mathrm{PNN}^{+}$, and $\mathrm{PV} / \mathrm{PNN}$ double-labelled cells. $\mathrm{N}$ $\mathrm{P} 14=29 / 29$, P28 $=53 / 52, \mathrm{P} 56=68 / 63$ images, four slices each from $\mathrm{P} 14=3 / 3, \mathrm{P} 28=3 / 3, \mathrm{P} 56=6 / 6 \mathrm{Ehmt}^{+/+} / \mathrm{Ehmt1}^{+/-}$animals. Colorcode per mouse. Source for a Allen Adult Mouse Brain Atlas, https ://atlas.brain-map.org/atlas?atlas $=1$. Image Credit: Allen Institute. Scale bars in b $500 \mu \mathrm{m}$, c, e $20 \mu \mathrm{m}$. Note that the intensities in c, e are matched within a timepoint, but not across timepoints. Scale in d, f cells $/ \mathrm{mm}^{2}$, Dot plot with mean \pm SEM, color-coded per mouse. $* p<0.05, * * p<0.01$; nested ANOVA with mice as subgroups. See Supplementary Table 1 for exact values

Slices were incubated for $1 \mathrm{~h}$ at $32{ }^{\circ} \mathrm{C}$, after which they were allowed to cool down to room temperature. The slices were then transferred to an upright microscope (Olympus) fitted with $2.5 \times$ and $40 \times$ water-immersion objectives and enhanced infrared illumination (DGC, Luigs \& Neumann), and incubated in "recording" ACSF $(124 \mathrm{mM} \mathrm{NaCl}, 10 \mathrm{mM}$ Glucose, $3 \mathrm{mM} \mathrm{KCl}, 1.25 \mathrm{mM} \mathrm{NaH}_{2} \mathrm{PO}_{4}, 2 \mathrm{mM} \mathrm{CaCl}_{2}$, $1 \mathrm{mM} \mathrm{MgCl} 2$, and $26 \mathrm{mM} \mathrm{NaHCO}_{3}$ ) at $30{ }^{\circ} \mathrm{C}$ with added $100 \mu \mathrm{M}$ D-AP5 (Tocris 0106) and 5.2 $\mu \mathrm{M}$ CNQX (Tocris 1045) to block NMDA and AMPA receptors, respectively.

For measuring miniature IPSCs (mIPSCs), slices were incubated in recording ACSF with $1 \mu \mathrm{M}$ TTX (Tocris 
a

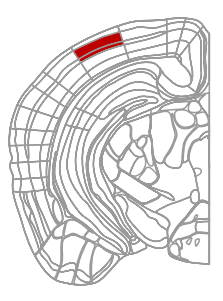

b

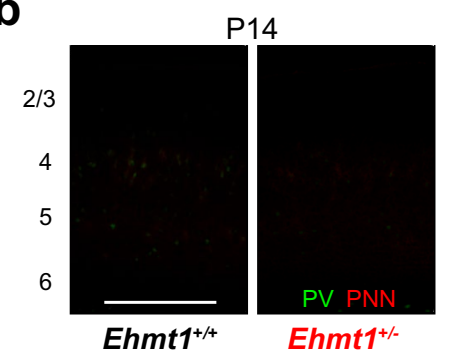

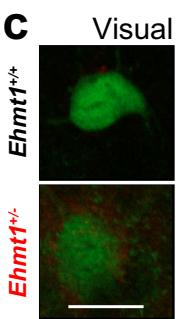

P14

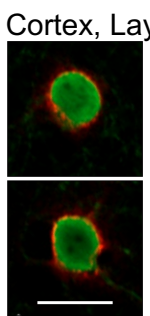

$\mathrm{P} 28$

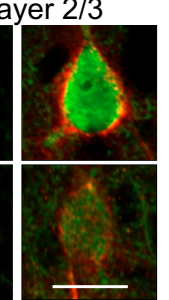

P56

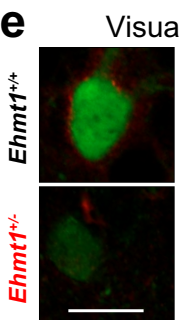

$\mathrm{P} 14$

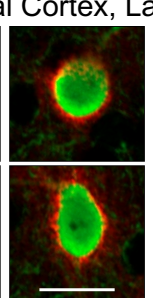

$\mathrm{P} 28$

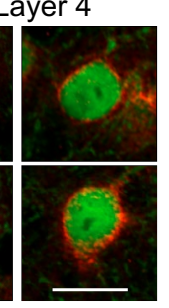

P56
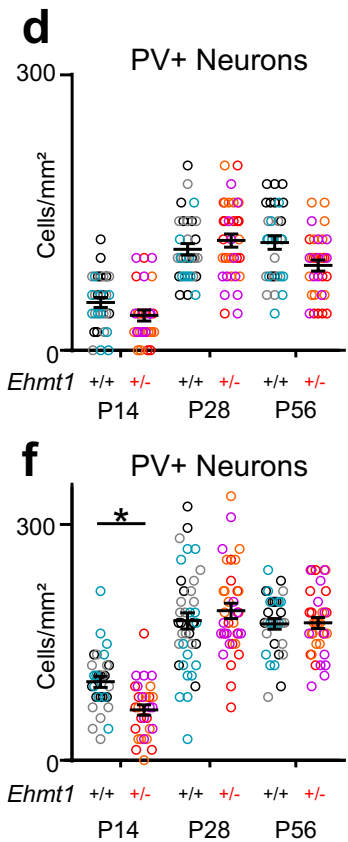

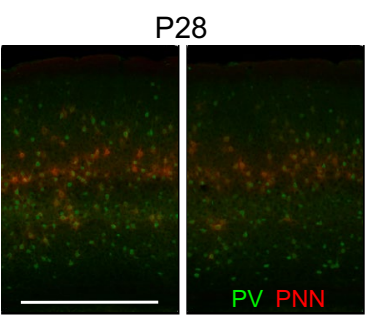

$E^{2}$ t1 $^{+/+}$

Ehmt1/-

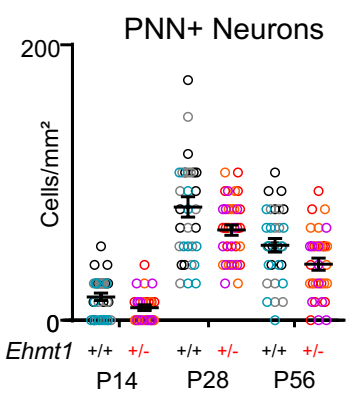

PNN+ Neurons

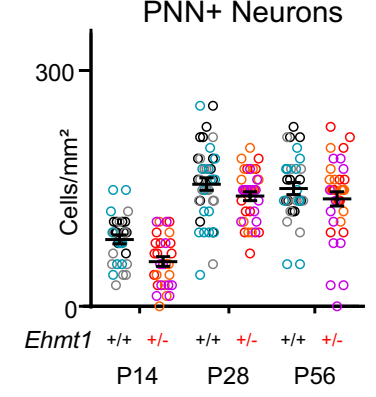

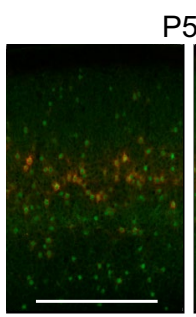

Ehmt1+/+ $^{++}$

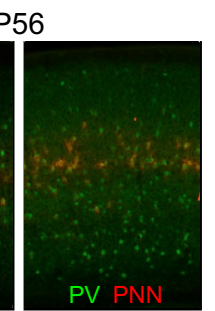

Ehmt1+- $^{+}$
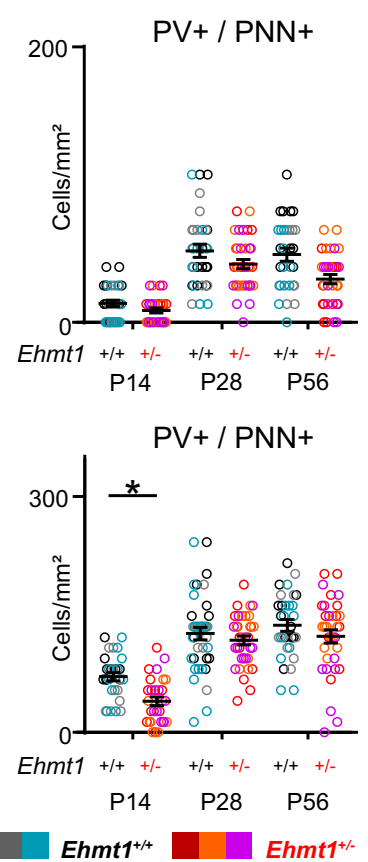

Fig. 3 An early delay in PV + maturation in Ehmt $^{+/-}$visual cortex layer 4 , but not layer 2/3. a Primary visual cortex, layers $2 / 3$ and 4 are marked in red. b Overview of $\mathrm{PV}^{+}$(green) and $\mathrm{PNN}^{+}$(red) development in the visual cortex over time. Layer numbers are marked on the left. c Neurons stained for Parvalbumin (green) and PNNs (red) in the primary Visual cortex, layer 2/3. d Quantification of the density of $\mathrm{PV}^{+}, \mathrm{PNN}^{+}$, and PV/PNN double-labelled cells. N P14=30/28, $\mathrm{P} 28=32 / 35$, P56=30/32 images, 12/12 slices from 3/3 Ehmtl ${ }^{+/+}$/ Ehmt $^{+/-}$animals per age. e Neurons stained for Parvalbumin (green) and PNNs (red) in the primary Visual cortex, layer 4. e, f Quantifica-

1069) to block action potentials, and $100 \mu \mathrm{M}$ D-AP5 (Tocris 0106) and 5.2 $\mu \mathrm{M}$ CNQX (Tocris 1045) to block excitatory transmission. Slices were allowed to adjust to the conditions for at least 10 min prior to patching. Pyramidal cells in auditory cortex layer $2 / 3$ were identified based on their perisomatic appearance at $40 \times$ magnification, and patched using 3-6 M $\Omega$ borosilicate pipettes filled with a Cesium-based intracellular solution containing $115 \mathrm{mM}$ $\mathrm{CsMeSO}_{3}, 20 \mathrm{mM} \mathrm{CsCl}$, $10 \mathrm{mM} \mathrm{HEPES,} 2.5 \mathrm{mM} \mathrm{MgCl}$, $4 \mathrm{mM}$ Na2ATP, $0.4 \mathrm{mM}$ NaGTP, $10 \mathrm{mM}$ Na-Phosphocreatine, $0.6 \mathrm{mM}$ EGTA, and $5 \mathrm{mM}$ QX-314 (Tocris 1014). Cells were patched at a minimum depth of $30 \mu \mathrm{m}$ from the tion of the density of $\mathrm{PV}^{+}, \mathrm{PNN}^{+}$, and PV/PNN double-labelled cells. N P14=30/29, P28=39/35, P56=31/34 images, 12/12 slices from 3/3 $\mathrm{Ehmt1}^{+/+} / \mathrm{Ehmt1}^{+/-}$animals per age. Source for a Allen Adult Mouse Brain Atlas, https://atlas.brain-map.org/atlas?atlas=1. Image Credit: Allen Institute. Scale bars in b $500 \mu \mathrm{m}$, c, e $20 \mu \mathrm{m}$. Note that the intensities in $\mathbf{c}$, e are matched within a timepoint, but not across timepoints. Scale in $\mathbf{d}, \mathbf{f}$ cells $/ \mathrm{mm}^{2}$, Dot plot with mean $\pm \mathrm{SEM}$, colorcoded per mouse. ${ }^{*} p<0.05$, ${ }^{*} p<0.01$; Nested ANOVA with mice as subgroups. Please see Supplementary Table 1 for exact values

slice surface to minimize cutting artefacts. The cells were held in voltage-Clamp mode controlled by an SEC 05-LX amplifier (NPI electronics, Tamm, Germany), low-pass filtered at $3 \mathrm{kHz}$ and sampled at $20 \mathrm{kHz}$ with a Power-1401 acquisition board and Signal software (CED, Cambridge, $\mathrm{UK}) . V_{\mathrm{h}}$ was adjusted to $+10 \mathrm{mV}$, and miniature inhibitory postsynaptic currents were recorded over 10 min per cell. Subsequently, the traces were exported from Signal as text files, saved as.abf with Clampfit and then loaded into MiniAnalysis. mIPSCs were identified manually, with an amplitude threshold of 8 pA, at least 300 events per cell. 
For measuring evoked GABA release (PPRs), slices were incubated in recording ACSF with $100 \mu \mathrm{M}$ D-AP5 (Tocris 0106) and 5.2 $\mu \mathrm{M}$ CNQX (Tocris 1045) added to block AMPA and NMDA-mediated currents, respectively. A tungsten bipolar stimulation electrode (CE2C55, FHC) coupled to an SD9 stimulator (Grass Instruments, RI, USA) or an ISO-STIM 01 M (NPI electronics, Tamm, Germany) was inserted into layer $2 / 3$ of the auditory cortex, and pyramidal cells were patched in layer $2 / 3$ next to the bipolar electrode $(<200 \mu \mathrm{m}$ lateral distance $)$ as described above. GABAergic currents were measured at a holding voltage of $-60 \mathrm{mV}$, and were completely abolished by application of Picrotoxin (data not shown). Stimulus duration (1-2 ms) and voltage were adjusted for a postsynaptic amplitude of $\sim 200 \mathrm{pA}$. For the paired-pulse ratio measurements, we measured a stimulus pair with 50,100, 150, 200, and $500 \mathrm{~ms}$ inter-stimulus interval (ISI), 5 sweeps per ISI, with 30 s pause between sweeps. We calculated the PPR as the ratio between peak amplitudes. For baseline 1, we used the average of the $500 \mathrm{~ms}$ before the first stimulus, and for baseline 2 we used the value directly before the second stimulus.

For the Agatoxin experiments, we patched pyramidal cells as described above, measured one PPR protocol at baseline (5 sweeps each of 5 ISIs, as above). Subsequently, we added $\omega$-Agatoxin-IVA (Sigma A6719) to a final concentration of $400 \mathrm{nM}$ (Zaitsev et al. 2007). GABAergic responses declined by ca. $85 \%$ over the following $10 \mathrm{~min}$ (Fig. S3), which was only the case with Agatoxin and not with control ACSF (data not shown). After 10 min incubation, we repeated the PPR protocol as described above.

For the high-Ca ${ }^{2+}$ incubations in Fig. $4 \mathrm{~g}-\mathrm{j}$, we added an additional $2 \mathrm{mM} \mathrm{Ca}^{2+}$ to the recording ACSF as described above, for a total ACSF $\mathrm{Ca}^{2+}$ concentration of $4 \mathrm{mM}$. The rundown experiments in Fig. 4i-k used 1-2 ms stimulus duration with initial peak amplitude adjusted to $\sim 200 \mathrm{pA}$. We used a train of 100 stimuli with $100 \mathrm{~ms}$ ISI $(10 \mathrm{~Hz})$, with a recovery stimulus at $500 \mathrm{~ms}$ after the end of the stimulus train and $30 \mathrm{~s}$ inter-sweep interval, for 10 sweeps in total. The trains were then exported from Signal as txt files, and amplitudes were quantified using a custom-written Python script. All further data processing was done in Excel. For the graph shown in Fig. 4i, we normalized all amplitudes to the first peak amplitude.

We calculated the readily releasable pool and the release probability by curve-fitting on a plot of the individual response amplitude against cumulative amplitude (Elmqvist and Quastel 1965; see for review Neher 2015). To get the RRP, normalized amplitudes ( $y$-axis) were plotted against normalized cumulative amplitudes ( $x$-axis). The steep initial change was extrapolated linearly, and the $x$-axis intercept gives the estimate of the readily releasable pool (RRP). The slope gives the estimate of the compound release probability $\left(P_{\mathrm{R}}\right)$.

\section{Statistics}

Data were processed in Excel and Python, and statistics and graphics were made in Excel, Python (Seaborn v0.10) and Graphpad Prism (version 7). Two-sample Kolmogorov-Smirnov tests for the mIPSC cumulative probability plots in Fig. 4b were done in RStudio. Unless otherwise indicated, the graphs show mean \pm SEM, and were tested for differences using either a nested ANOVA using mice as subgroups and genotypes as groups, or two-sided Student's $t$ test with Bonferroni correction for multiple testing.

\section{Results}

We first investigated whether a heterozygous loss of Ehmt 1 influences the developmental trajectory of $\mathrm{PV}^{+} \mathrm{GABAergic}$ interneurons in the sensory cortices in mice. We compared heterozygous Ehmtl mutant $\left(E h m t 1^{+/-}\right)$mice to wild-type $\left(E h m t 1^{+/+}\right)$littermates, a construct valid model for KS (Balemans et al. 2010), and because a full knockout is lethal at the embryonic stage (Tachibana et al. 2005). We selected three well-characterized sensory regions: primary auditory cortex (A1), the barrel subfield in the primary somatosensory cortex (S1), and the primary visual cortex (V1). In each region, we generated a timeline of $\mathrm{PV}^{+}$neuron maturation, measuring two maturation markers, Parvalbumin (PV) and Perineuronal Nets (PNNs) in cortical layers $2 / 3$ and 4 . Both layers are crucial for sensory cortex function. In the simplest version of the canonical sensory cortical model, layer 4 is the thalamo-recipient "input" layer that first receives sensory input during development. Layer 4 neurons then send the processed input to neurons in layer $2 / 3$, which integrate both locally and wide-range inputs. Consequentially, layer $2 / 3$ is thought to mature later than layer 4 (Butt et al. 2017). We selected three timepoints based on the developmental trajectory of the primary auditory cortex (A1): P14 is expected to be the onset of the auditory critical period for thalamocortical input to layer 4 (Barkat et al. 2011). It is also the timepoint at which Ehmt1 ${ }^{+/+}$mice show an acoustic startle response (Balemans et al. 2014). The second timepoint, P28, is thought to be after the end of the auditory cortical critical period (Oswald and Reyes 2011; Kalish et al. 2020). For the third timepoint we selected P56, when mice are considered adult for the purposes of sensory development.

\section{Expression of PV and PNNs is delayed In Ehmt ${ }^{+/-}$ primary auditory cortex}

We first measured the developmental trajectory of $\mathrm{PV}^{+}$neurons in the primary auditory cortex, in both layers $2 / 3$ and 4 (Fig. 1a). We visualized PV by immunostaining and PNN by staining with Wisteria Floribunda Agglutinin (WFA), and 

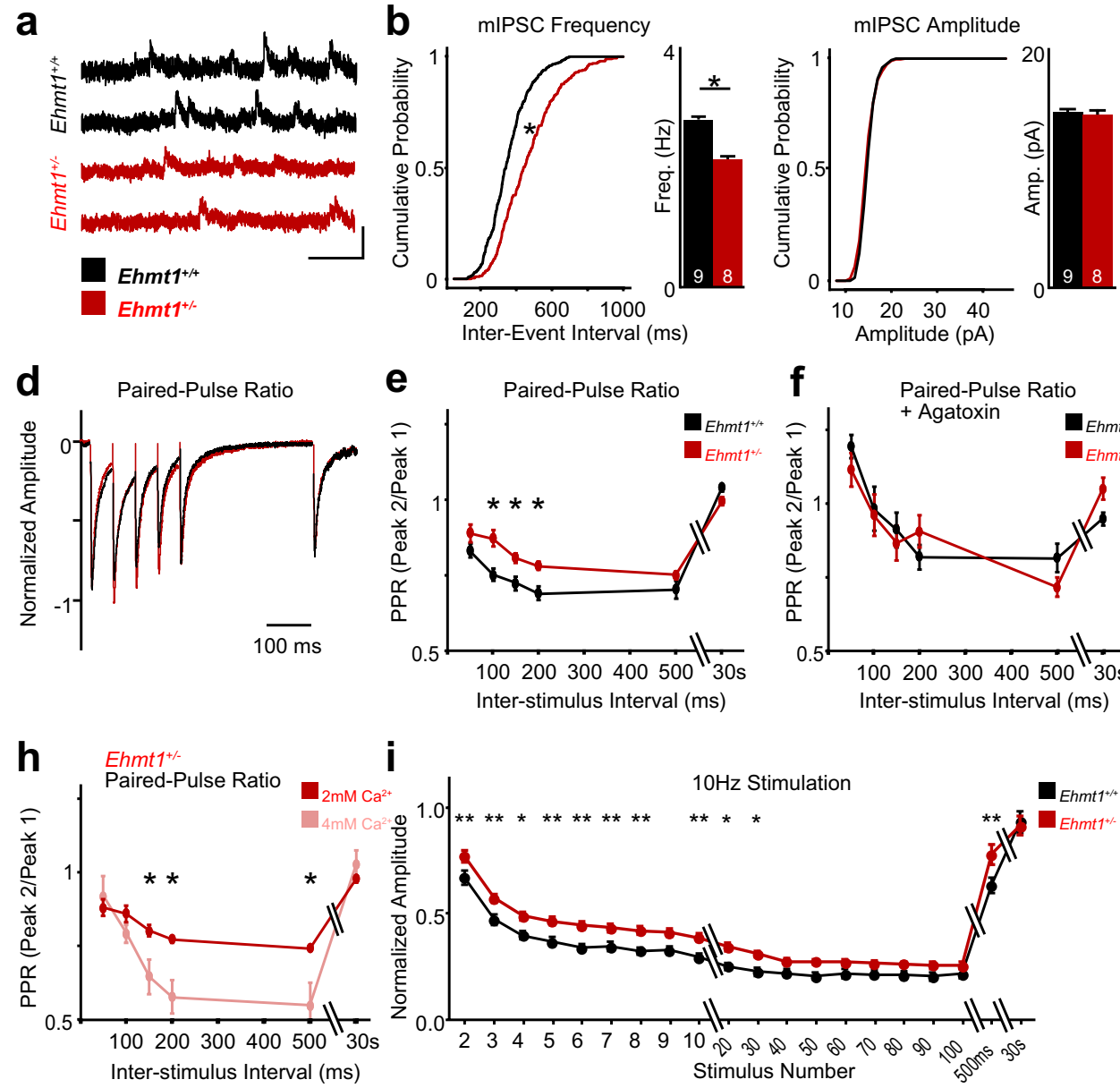
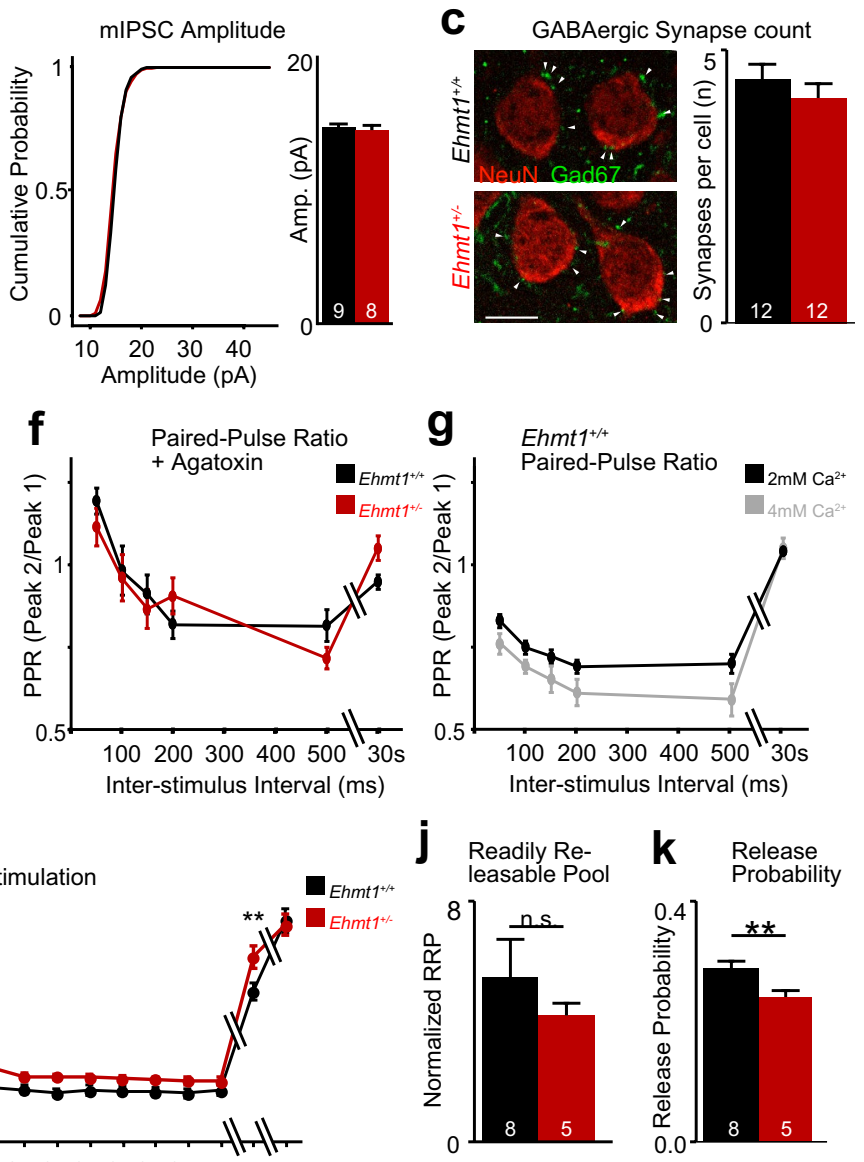

Fig. 4 Inhibitory GABAergic transmission is impaired in Ehmt ${ }^{+/-}$ auditory cortex at P14-16. a Representative traces of inhibitory miniature postsynaptic currents (mIPSCs) recorded from pyramidal neurons in $E h m t 1^{+/+}$(black) and Ehmt1 ${ }^{+/-}$(red). Scale bar $=20 \mathrm{pA}$, $100 \mathrm{~ms}$. b Cumulative probability plots of mIPSC Frequency (left), and mIPSC amplitude (right). $N=9 / 8$ cells. c Left, Putative inhibitory presynapses (arrowheads) stained with Gad67 (green) next to neuronal somata (NeuN, red). Right, quantification of perisomatic Gad67 $7^{+}$puncta. $N=319 / 237$ cells in $12 / 12$ slices from 3/3 Ehmt ${ }^{+/+}$/ Ehmt $^{+/-}$mice. Student's $t$ test. d Composite traces illustrating the paired-pulse paradigm. GABAergic inputs to A1 L2/3 pyramidal cells are stimulated at intervals of respectively 50,100,150,200, or $500 \mathrm{~ms}$. Scale bar $=100 \mathrm{~ms}$. e Paired-pulse ratio for $E h m t 1^{+/+}$(black) and EhmtI $^{+/-}$(red). $N=14 / 14$ cells from 4/4 Ehmt ${ }^{+/+} /$Ehmt $^{+/-}$ani-

counted the presence of $\mathrm{PV}^{+}, \mathrm{PNN}^{+}$and $\mathrm{PV} / \mathrm{PNN}$ doublelabelled cells in both layers $2 / 3$ and 4 . Consistent with an ongoing developmental process, in $\mathrm{Ehmt}^{+/+}$the density of both $\mathrm{PV}^{+}$and $\mathrm{PNN}^{+}$cells increased from P14 to P28 and levelled off afterwards, and was higher in layer 4 compared to layer 2/3 (Fig. 1b). We found a significant reduction in the density of $\mathrm{PV}^{+}$and $\mathrm{PNN}^{+}$neurons at $\mathrm{P} 14$, both in layer $2 / 3$ (Fig. 1 c-d) and layer 4 (Fig. 1e, f, Supplementary Fig. S1, Supplementary Table S1) in Ehmt ${ }^{+/-}$compared to Ehmt $1^{+/+}$ mice. Consequentially, we also found a reduction in PV/ PNN double-labelled cells in both layers at P14. After P14, mals. f Paired-pulse ratio in the presence of $\omega$-Agatoxin-IVA, a specific blocker of $\mathrm{P} / \mathrm{Q}$ type $\mathrm{Ca}^{2+}$ channels. g Comparison of $E h m t 1^{+/+}$ PPR at physiological (2 $\mathrm{mM}$, black) and elevated (4 mM, grey) extracellular $\mathrm{Ca}^{2+}$. h Comparison of $\mathrm{Ehmtl}^{+/-} \mathrm{PPR}$ at physiological ( $2 \mathrm{mM}$, red) and elevated (4 mM, salmon) extracellular $\mathrm{Ca}^{2+}$. i Rundown of vesicle pools by $10 \mathrm{~Hz}$ stimulation at $4 \mathrm{mM}$ extracellular $\mathrm{Ca}^{2+}$. Normalized amplitudes plotted against time; 100 stimuli with $100 \mathrm{~ms}$ ISI, followed by control pulses at $500 \mathrm{~ms}$ and $30 \mathrm{~s}$ (sweep end). Multiple $t$ tests with Benjamini-Hochberg correction. j Readily releasable pool determined from the $10 \mathrm{~Hz}$ stimulus train. RRP displayed normalized to the initial stimulus amplitude. $\mathbf{k}$ Release Probability determined from the $10 \mathrm{~Hz}$ stimulus train. ${ }^{*} p<0.05$, $* * p<0.01$. Please see Supplementary Table 2 for exact values

all measures normalized to $\mathrm{Ehmtl}^{+/+}$levels in both layer 2/3 (Fig. 1c, d) and layer 4 (Fig. 1e, f). The density of $\mathrm{PV}^{+}$neurons in layer 4 was at a slightly lower level at P56 compared to P28 (Fig. 1f), which might indicate a dynamic downregulation as observed between P30-40 (Bhumika et al. 2020). This downregulation in layer 4 appears to be unchanged between genotypes, as we did find the same distribution at P56 both with slice immunostainings (Fig. 1f) and genetic labelling ( $\mathrm{PV}^{\mathrm{Cre}}$-Tdtomato ${ }^{\text {flox }}$, Supplementary Fig. S2c). These findings indicate that initial $\mathrm{PV}^{+}$neuron maturation is delayed in $\mathrm{Ehmtl}^{+/-}$auditory cortex. Interestingly, P14 
marks the beginning of the formation of the tonotopic representation in the auditory cortex (Oswald and Reyes 2011), and thus the beginning of the auditory critical period (Barkat et al. 2011; Kalish et al. 2020). In agreement with a delayed auditory cortical development, Ehmt ${ }^{+/-}$mice also display a delayed acoustic startle response around this time $\left(E h m t 1^{+/+}\right.$ P13.5, Ehmt1 ${ }^{+/-}$P14.5; Balemans et al. 2014).

\section{The Ehmt 1/-- somatosensory cortex shows a consistent reduction of $\mathrm{PV}^{+}$cell markers in layer 4 , but not in layer $2 / 3$}

The somatosensory cortex is the earliest sensory cortex to mature, with input to layer 4 maturing by between P5 and $\mathrm{P} 8$, and layer $2 / 3$ between $\mathrm{P} 10$ and $\mathrm{P} 15$, and mature $\mathrm{PV}^{+}$ function by P12 (Butt et al. 2017). The timepoints map onto late critical period for layer $2 / 3$ (P14), post-critical period (P28) and adulthood (P56). As in the auditory cortex, we observed the largest density of PV/PNN labelled neurons in layer 4 , but in contrast to auditory cortex, we also observed a double-labelled population in layer $5 b$ (Fig. 2b). When quantifying the PV/PNN-labelled neurons in layer $2 / 3$, we found no change at any timepoint (Fig. 2c, d, Supp. Table S1), except a lower percentage of $\mathrm{PV}^{+}$neurons with PNNs at P14 (Supplementary Fig. S1). This matches with P14 being in the middle of the critical period for input to layer 2/3 (Butt et al. 2017).

In the Ehmt1 ${ }^{+/-}$layer 4 however, we found that all three measures were significantly reduced compared to $E h m t 1^{+/+}$. Interestingly, this reduction in $E$ hmt ${ }^{+/-}$persisted throughout P28 and into adulthood at P56 (Fig. 2e, f, Supplementary Table S1), with the exception of $\mathrm{PV}^{+}$at $\mathrm{P} 28$. We furthermore found an especially large reduction in $\mathrm{PV}^{+}$and $\mathrm{PV} /$ PNN double-labelled neuron density in $\mathrm{Ehmtl}^{+/-}$at P56 (Fig. 2f), indicative of an ongoing reduced $\mathrm{PV}^{+}$expression, which does not happen in wild-type mice, even at advanced ages (Ueno et al. 2019). Thus, in contrast to all other areas studied, in the $\mathrm{Ehmtl}^{+/-}$somatosensory cortex layer 4 we find a persistent $\mathrm{PV}^{+}$neuron maturation deficit. This could be either due to a reduced density of $\mathrm{PV}^{+}$precursor neurons (i.e. generation or migration of MGE-derived neurons), or due to a dynamic downregulation of $\mathrm{PV}^{+}$expression. In order to differentiate between both options, we genetically labelled neurons in which the $\mathrm{PV}^{+}$promoter had been active at any time $\left(\mathrm{PV}^{\mathrm{Cre}}-\right.$-Tdtomato ${ }^{\text {flox }}$, Supplementary Figure S2a). Following whole-brain immunostaining and clearing with the iDISCO + protocol (Renier et al. 2014, 2016), we found that the $\mathrm{PV}^{+}$neuron distribution was similar between $\mathrm{Ehmtl}^{+/-}$and $\mathrm{Ehmtl}^{+/+}$at P56 in S1, A1, or V1 (Supplementary Figure S2c). This indicates a continuous downregulation of $\mathrm{PV}$ in $\mathrm{PV}^{+}$neurons in the $E h m t 1^{+/-} \mathrm{S} 1$, possibly already at our earliest timepoint $\mathrm{P} 14$, which is after the critical period for somatosensory cortex layer 4
(Butt et al. 2017). As a consequence, the $\mathrm{E} / \mathrm{I}$ balance in the Ehmt ${ }^{+/-}$somatosensory cortex could be shifted towards over-excitability, which would predict a deficit in whiskerbased sensation. This prediction is consistent with previous behavioral studies that indicate a reduction in exploration behavior and neophobia (Balemans et al. 2010, 2014).

\section{$\mathrm{PV}^{+}$maturation is reduced early in Ehmt $1^{+/-}$Visual cortex layer 4}

The visual cortex starts receiving sensory input after eye opening around $\mathrm{P} 13$, and therefore is the last primary sensory cortex to mature (Espinosa and Stryker 2012). Its critical period is typically defined as the refinement of binocular representations, which is thought to start after P21 and continue until at least $\mathrm{P} 35$, with some plasticity remaining long afterwards (Espinosa and Stryker 2012; Hensch 2005).

From all tested areas, the visual cortex showed the lowest overall count of neurons in both layer $2 / 3$ and 4 at P14 (Fig. 3b), consistent with a time period in which incoming sensory input is only starting to trigger PV expression. When we quantified the density of PV/PNN labelled neurons in layer 4, we found a significant reduction of $\mathrm{PV}^{+}$and $\mathrm{PV} /$ PNN double-labelled neuron density at P14, comparable to layer 4 in A1 and S1 (Fig. 3e, f). Similar to A1 layer 4, both $\mathrm{PV}^{+}$and PV/PNN double-labelled densities caught up to Ehmt $^{+/+}$levels by P28 (Fig. 3e, f), and at adulthood both genotypes were indistinguishable both in slice stainings (Fig. 3e, f) and genetically labelled $\mathrm{PV}^{+}$neurons (Supplementary Fig. S2c).

The phenotype we found in V1 layer 4 is thus comparable to the ones found in the other sensory regions at P14. In the case of the visual cortex, a difference in the arrival of sensory input is readily apparent, where $E h m t 1^{+/-}$mice open their eyes on average at P16, approximately 2 days later than Ehmt $^{+/+}$mice (Balemans et al. 2014). Consequentially, the Ehmt $\mathrm{I}^{+/-}$mice measured at P14 would on average still have had their eyes closed, and therefore received less visual input than their WT littermates. Interestingly, our findings indicate that $\mathrm{Ehmtl}^{+/-}$visual cortex $\mathrm{PV}^{+}$neurons were maturing similarly to WT neurons by the onset of the visual cortex critical period (P21-35).

We then wondered whether the density reduction we observed in $\mathrm{EhmtI}^{+/-}$mice was similar across mice, or whether each $E h m t 1^{+/-}$mouse shows a reduction only in a subset of areas. In order to assess inter-individual variability, we normalized the densities of each $\mathrm{EhmtI}^{+/-}$mouse to the average of the $E h m t 1^{+/+}$mice. We plotted the $\mathrm{PV}^{+} / \mathrm{PNN}^{+}$ results per $E h m t 1^{+/-}$mouse for which we had data from all areas (Supplementary Fig. S3, Supplementary Table S1). Our findings largely mirrored the findings in Figs. 1, 2 and 3. Specifically, a general trend in the $E h m t l^{+/-}$towards lower values than $E h m t 1^{+/+}$across all timepoints, with the most 
pronounced difference at P14 across all areas, and more subtle differences afterwards. Accordingly, when comparing the variation between genotypes, we observed a trend towards higher Coefficient of Variation in Ehmtl ${ }^{+/-}$at P14, but not at P28 and P56 (Supplementary Table S1). Interestingly, those differences in $\mathrm{CV}$ at P14 appear to be driven mainly by layers $2 / 3$ rather than layers 4 . However, the normalized densities per mouse (Supplementary Fig. S3) suggest that the reductions in $E h m t 1^{+/-}$appear to be similar across all measured areas per mouse, rather than each mouse exhibiting reductions in a subset of areas.

\section{Reduced inhibitory synaptic transmission in $\mathrm{Ehm} \mathrm{II}^{+/-}$is caused by reduced GABA release from putative $\mathrm{PV}^{+}$neurons}

Next, we wondered whether the delay in $\mathrm{PV}^{+}$neuron maturation at P14 would lead to reduced GABAergic transmission onto pyramidal neurons. To this end, we measured miniature inhibitory postsynaptic currents (mIPSCs, Fig. 4a, b) in acute slices of the auditory cortex layer $2 / 3$ at P14-16. Inhibitory inputs are likely to be dominated by inhibitory inputs from basket cells, most of which are fast-spiking $\mathrm{PV}^{+}$ neurons (Tremblay et al. 2016). However, as we have shown in Fig. 1, PV expression is still being established at P14 in A1 layer 2/3, so even though basket cells are present and provide GABAergic input, only a subset of the basket cell population will already express $\mathrm{PV}$.

We found a significant reduction in MIPSC frequency in Ehmt $^{+/-}$neurons, but no change in amplitude (Fig. 4a, b, Supp. Table S2). Interestingly, the reduced mIPSC frequency was not apparent when recorded at P21, after the closure of the auditory critical period in normally developing mice (Supplementary Figure S5). The reduced mIPSC frequency could be explained either by a reduced number of synapses or a reduced release probability. To distinguish between both possibilities, we first stained for perisomatic GABAergic presynapses on pyramidal cells, which mostly originate from basket-cells (present or future $\mathrm{PV}^{+}$). We found no difference in the number of GABAergic $\left(\mathrm{Gad}_{6} 7^{+}\right)$puncta between genotypes (Fig. 4c), suggesting that the change in mIPSC frequency we observed is not caused by a reduced perisomatic synapse number, but rather by a reduced release probability.

Next, to test whether the GABAergic release probability is reduced, we measured the paired-pulse ratio (PPR) for GABAergic input onto pyramidal neurons (Fig. 4d, e, Supp. Table S2). PPR for input from $\mathrm{PV}^{+}$neurons has been shown to be depressing, i.e. the response to the second stimulus is lower than the response to the first stimulus (Chang et al. 2014). We did indeed find a rapidly depressing paired-pulse response in $\mathrm{Ehmtl}^{+/+}$slices (Fig. 4d, e). However, we found the PPR to be less depressing in the Ehmt1 ${ }^{+/}$, specifically at $100 \mathrm{~ms}, 150 \mathrm{~ms}$, and $200 \mathrm{~ms}$ inter-stimulus interval (Fig. 4d, e), indicating that GABAergic release is affected in $\mathrm{Ehmt1}^{+/-}$mice.

Next, we wondered whether the less-depressing PPR in Ehmt1 ${ }^{+/-}$is specific to the synapses originating from $\mathrm{PV}^{+}$ neurons. To this end, we blocked GABA release specifically from $\mathrm{PV}^{+}$neurons by blocking their dominant $\mathrm{Ca}^{2+}$ channel (P/Q channels) via bath application of $\omega$-Agatoxin-IVA (400 nM, Zaitsev et al. 2007). After $\omega$-Agatoxin-IVA application, the remaining IPSC amplitude was reduced by approx. $85 \%$, and remaining IPSC decay time was slower (Supplementary Figure S6), indicating that $\omega$-Agatoxin-IVA blocked fast inhibitory transmission from $\mathrm{PV}^{+}$neurons, in agreement with previous reports in the rat prefrontal cortex (Zaitsev et al. 2007). More importantly, following $\omega$-Agatoxin-IVA application, both Ehmt ${ }^{+/+}$and Ehmt ${ }^{+/-}$showed similar PPRs (Fig. 4f, Supplementary Table S2), indicating that the PPR phenotype in $\mathrm{Ehmtl}^{+/-}$without $\omega$-Agatoxin-IVA originates from $\mathrm{PV}^{+}$neurons.

We further wondered whether the less-depressing PPR in $E h m t 1^{+/-}$was caused by a reduced release probability $\left(P_{\mathrm{R}}\right)$, or by a change in the vesicle pool available for release (readily releasable pool). To this end, we measured PPRs with elevated $P_{\mathrm{R}}$ by elevating the extracellular calcium level to $4 \mathrm{mM} \mathrm{Ca}^{2+}$. We found similar PPRs in $\mathrm{EhmtI}^{+/+}$ under baseline $\left(2 \mathrm{mM} \mathrm{Ca}^{2+}\right)$ and elevated $\mathrm{Ca}^{2+}\left(4 \mathrm{mM} \mathrm{Ca}^{2+}\right)$ conditions (Fig. 4g, Supplementary Table S2). This might indicate a ceiling effect for GABAergic release in the wildtype condition (see for a review: Neher 2015). In contrast, we found that $E h m t 1^{+/-}$neurons showed significantly more paired-pulse depression at $4 \mathrm{mM} \mathrm{Ca}^{2+}$ than at $2 \mathrm{mM} \mathrm{Ca}^{2+}$, specifically at 150, 200, and $500 \mathrm{~ms}$ ISI (Fig. 4h, Supplementary Table S2), which implies that $E h m t 1^{+/-}$mice might have a lower baseline release probability.

In order to fully quantify the release probability and readily releasable pool, we exhausted the synaptic pools with a depletion protocol consisting of trains of 100 stimuli at $10 \mathrm{~Hz}$ at elevated $\mathrm{Ca}^{2+}$ levels $(4 \mathrm{mM}$; Fig. 4i). In both genotypes, we observed a rapid rundown of GABAergic responses, eventually reaching a steady state. However, in $E h m t^{+/-}$, the relative amplitudes during the rundown were higher than in Ehmtl ${ }^{+/+}$(Fig. 4i). We hypothesized that this finding could be consistent with a reduced release probability, but not necessarily a smaller vesicle pool. Specifically, if a smaller portion of the pool would get released with each subsequent pulse, then reaching the steady-state level would take more stimulations.

We therefore estimated both the readily releasable pool and release probability from the $10 \mathrm{~Hz}$ stimulus trains. Consistent with our hypothesis, we found that the readily releasable pool was unchanged between $E h m t 1^{+/+}$and $E h m t 1^{+/-}$at an estimated 7 initial stimulus amplitude sizes (Fig. 4j, Supplementary Table S2). The release probability, in contrast, 
was indeed significantly lower in Ehmt1 ${ }^{+/-}$(Fig. 4k, Supplementary Table S2), confirming our hypothesis and pinpointing the impaired GABAergic transmission in $\mathrm{Ehmtl}^{+/-}$cortex to $\mathrm{PV}^{+}$presynapses.

To conclude, our electrophysiological experiments paint a consistent picture of a reduced GABA release probability in Ehmt ${ }^{+/-}$A1 layer 2/3 at P14, specific to putative $\mathrm{PV}^{+}$ synapses. This synaptic deficit matches the delay in $\mathrm{PV}^{+}$ neuron maturation observed in the same region and time (A1 layer 2/3 at P14; Fig. 1c, d) and indicates an impairment in both maturation and function of $\mathrm{PV}^{+}$neurons in the early sensory cortices of $\mathrm{EhmtI}^{+/-}$mice.

\section{Discussion}

Here we describe a region- and time point-specific influence of Ehmtl haploinsufficiency on the development and function of $\mathrm{PV}^{+}$neurons. In all three sensory cortical areas, we found a reduction of $\mathrm{PV}^{+}$developmental markers in layer 4 at P14 (see Supplementary Fig. S4 for an overview). In Ehmtl $^{+/-}$A1 and V1, those markers normalized to wildtype levels by $\mathrm{P} 28$, indicating a delayed $\mathrm{PV}^{+}$maturation. In contrast, in the Ehmt1 ${ }^{+/-} \mathrm{S} 1$ layer $4, \mathrm{PV}^{+}$maturation markers remained reduced into adulthood (P56), which implies an area-specific permanent immature state for $\mathrm{S} 1$ in Ehmt1 ${ }^{+/-}$mice. Genetic labelling coupled with whole-brain immunostaining and clearing using the iDisco + protocol (Renier et al. 2014, 2016) showed no difference between the genotypes at P56 in any primary sensory cortex (Supplementary Fig. S2), including S1 layer 4. This indicates that in Ehmtl ${ }^{+-}$mice, the generation and migration of MGEderived (future $\mathrm{PV}^{+}$and $\mathrm{SST}^{+}$neurons) is not impaired in the primary sensory cortices, which raises the interesting possibility that continuous downregulation of $\mathrm{PV}^{+}$neurons identified with anti-PV immunostainings might be an activity-dependent downregulation due to reduced circuit activity in the barrel cortex.

The "integrative" layer $2 / 3$ was more variable across regions and time. In the A1 layer 2/3, we observed a developmental delay in $E h m t 1^{+/-}$, similar to A1 layer 4. However, S1 and $\mathrm{V} 1$ layer $2 / 3$ showed no differences between genotypes at any age (Supplementary Fig. S4). We then investigated whether the immature $\mathrm{PV}^{+}$phenotype at $\mathrm{P} 14$ is also reflected at the functional level. We found that GABAergic input to A1 layer 2/3 pyramidal neurons is reduced in $E h m t 1^{+-}$. This reduction is selectively caused by a reduced GABA-release probability at the $\mathrm{PV}^{+}$presynapse in $E h m \mathrm{HI}^{+/-}$cortex.

Auditory cortex is the only sensory area we tested where $\mathrm{PV}^{+}$maturation was impaired in both $\mathrm{L} 2 / 3$ and 4 at $\mathrm{P} 14$, but normalized later in development. In mice, P14 is the middle of the critical period for the auditory cortical tonotopic map (Barkat et al. 2011; Takesian et al. 2018). It therefore seems that in $\mathrm{Ehmt}^{+/-}$mice, the auditory critical period might be shifted past P14, which is consistent with earlier studies in which the appearance of an acoustic startle response was delayed in $E h m t 1^{+/-}$by approximately one day, from P13.5 in Ehmtl ${ }^{+/+}$to P14.5 in Ehmt1 ${ }^{+/-}$(Balemans et al. 2014).

This poses the question whether an early change in $\mathrm{PV}^{+}$ developmental trajectory causes lasting network defects in Ehmt1 ${ }^{+/-}$sensory cortices, even once the expression of PV and PNNs have normalized to wild-type levels. Evidence from other mouse models with changed $\mathrm{PV}^{+}$developmental trajectories indicates that this could be the case. In Mecp $2^{-/ y}$ mice, for example, $\mathrm{PV}^{+}$neurons develop precociously before the onset of vision, resulting in impaired sensory perception in adulthood (Durand et al. 2012; Patrizi et al. 2019) and a premature closure of the critical period (Krishnan et al. 2015). An early sensory change affects sensorycortex dependent learning in adult mice, recently shown in $M e c p 2^{+/-}$mice with pup retrieval, a learned maternal behavior that is dependent on active remodeling of $\mathrm{PV}^{+}$neurons (Krishnan et al. 2017). Specifically, Mecp $2^{+/-}$females showed impaired PV and PNN remodeling following surrogate maternal experience, and consequentially displayed impaired pup-gathering behavior (Krishnan et al. 2017; Lau et al. 2020a, b).

In the primary visual cortex, we observe an early (P14) $\mathrm{PV}^{+}$maturation delay in $\mathrm{Ehmt}^{+/-}$layer 4, but not in layer 2/3. P14 is just after the timepoint of eye opening in WT mice, and this event is delayed in Ehmt ${ }^{+/-}$mice, to P16 (Balemans et al. 2014). As a result, the reduced $\mathrm{PV}^{+}$ interneuron count in layer 4 at P14 could be due to a delay in the arrival of sensory information in Ehmt ${ }^{+/-}$mice. Interestingly, a delayed onset of visual perception has been linked to a delayed closure of the auditory critical period in wildtype mice (Mowery et al. 2016), which poses an interesting possibility of cross-modal plasticity.

In the $\mathrm{Ehmtl}^{+/-}$somatosensory cortex layer 4, we found that the density of $\mathrm{PV}^{+}$interneurons remains low into adulthood. A possible explanation might be a blurred representation of the barrel field, as for example in $\mathrm{Fmrl}^{-/ y}$ mice (Juczewski et al. 2016). As $\mathrm{PV}^{+}$interneurons are located in the barrel walls, a blurred representation might imply a permanently reduced $\mathrm{PV}^{+}$interneuron count as well (Selby et al. 2007). Alternatively, the reduced $\mathrm{PV}^{+}$count might reflect an inactive state of the whisker system in general, similar to a state of sensory deprivation (McRae et al. 2007), which would be consistent with the fact that genetic labelling of $\mathrm{PV}^{+}$neurons did not show a difference between genotypes (Supp. Fig. S2c). The somatosensory system is the only of the sensory systems tested here that requires active movement (i.e. sweeping of the whiskers), with precisely timed sensorimotor integration between S1, secondary somatosensory cortex (S2) and motor cortical areas (see for a review: Petersen 2007). This type of precise, long-range connectivity 
between areas may be impaired in $E h m t I^{+/-}$mice, for example due to dysregulated expression of guidance cues such as clustered Protocadherins (Iacono et al. 2018). Defects may also occur further upstream, as Ehmtl is also expressed in the developing whisker pad (Kleefstra et al. 2005) and during the development of sensory subcortical circuitry (Ebbers et al. 2016). Functionally, a permanently reduced $\mathrm{PV}^{+}$interneuron activity such as caused by early whisker trimming has been linked to deficits in sensory acuity, hypersensitivity to stimuli (Antoine et al. 2019) and social deficits (Wolfe et al. 2011). As a consequence, the deficits in social and sensory behavior in Ehmt ${ }^{+/-}$mice may be partially mediated by a reduced S1 L4 inhibitory function (Balemans et al. 2010, 2013, 2014).

The emergence of PV and PNNs was approximately equal in our measurements, which indicates that PV and PNN are expressed at approximately the same rate (Supplementary Fig. S3). When we plot the percentage of $\mathrm{PV}^{+}$cells that also express PNNs (Supplementary Fig. S1), we see that the percentage of double-labelled $\mathrm{PV}^{+}$neurons is generally higher in layer 4 than layer $2 / 3$, and higher in the somatosensory cortex than in either visual or auditory. Interestingly, we only observe significant reductions in the percentage of doublelabelled cells in $\mathrm{Ehmtl}^{+/-}$during the critical period for the respective region, with $\mathrm{A} 1$ layer $2 / 3$ and 4 at P14, S1 layer 2/3 at P14, and V layer 2/3 at P28 (Supplementary Figure S1). PNNs are honeycomb-like structures that enwrap soma and proximal dendrites of (mostly) $\mathrm{PV}^{+}$neurons (review: Testa et al. 2019). Recent reports indicate the activity level of $\mathrm{PV}^{+}$neurons cell-autonomously regulate the production of PNNs and PNN-modifying enzymes even in adulthood (Devienne et al. 2019). In the $F m r l^{-/ y}$ mouse, a delay of both $\mathrm{PV}^{+}$and PNN development is visible, which in this mouse is caused by an overproduction of the PNN modifier MMP-9 during the auditory critical period (Wen et al. 2018). Interestingly, while the $\mathrm{PV}^{+}$developmental trajectory in $\mathrm{Fmrl}^{-/ y}$ matches our Ehmtl ${ }^{+/-}$findings, MMP-9 is downregulated during development in the hmtl $^{+/-}$cortex (Iacono et al. 2017). Thus, here we observe a phenotypic convergence despite molecular divergence in the PNN modification pathway.

PNNs enhance the capacity of $\mathrm{PV}^{+}$neurons for highfrequency firing by reducing the membrane capacitance (Tewari et al. 2018) and clustering ion channels (Favuzzi et al. 2017). PNNs also bind extracellular molecules that are important for the developmental trajectory of $\mathrm{PV}^{+}$neurons, for example the axon guidance cues Semaphorin $3 \mathrm{a} / \mathrm{b}$ (De Winter et al. 2016) and the trans-synaptic transcription factor Otx2. This homeoprotein is not produced anywhere in the cortex, but rather in sensory afferents and the choroid plexus (Spatazza et al. 2013; Sugiyama et al. 2008). Otx2 binds to PNNs and enters the future $\mathrm{PV}^{+}$cell, where it triggers a genetic program for critical period plasticity
(Beurdeley et al. 2012; Prochiantz and Di Nardo 2015; Testa et al. 2019) by upregulation of Gadd45b, a DNA demethylase that orchestrates the large-scale change in the transcriptomic landscape during the critical period, including clustering of MeCP2 in the nucleus (Apulei et al. 2019), where it has been shown to directly bind to and methylate the Pvalb promoter region (Patrizi et al. 2019). Interestingly, $M e C P 2$ has recently been shown to be a direct target of Ehmtl in non-neuronal cells (Choi et al. 2018). Besides signalling by Otx2, another interesting candidate might be the signalling molecule BDNF, which is both important for $\mathrm{PV}^{+}$neuron maturation (Berghuis et al. 2006; Huang et al. 1999) and negatively regulated by EHMT1 (Benevento et al. 2016). Specifically, EHMT1 methylates histones around the activity-dependent $B d n f$ promoter IV in response to network silencing, leading to a failure of activity-dependent $B d n f$ repression in $\mathrm{Ehmt}^{+/-}$neurons (Benevento et al. 2016). However, it is currently unknown whether EHMT1 also plays a role in $B d n f$ upregulation via promoters other than $B \operatorname{dnf} I V$, as happens during normal cortical development (Du et al. 2018). Interestingly, emerging evidence links BDNF signaling to the $M e C P 2^{-/ y}$ synaptic phenotype as well (Sampathkumar et al. 2016), even though also in this case a precise molecular mechanism still remains to be found. Quite possibly, several different signaling systems (e.g. Otx2 and BDNF, as well as circuit activity levels) could converge on $\mathrm{PV}^{+}$neurons during the critical period, triggering a common genetic program including PV expression and PNN production. In turn, the actual execution of this of this genetic program would require precisely targeted epigenetic modulation, possibly including Gadd45b and MeCP2 as well as EHMT1. Consequentially, an Ehmt1 haploinsufficiency could cause an incomplete transition from the pre-critical period transcriptomic state to the post-critical period transcriptomic state, thus delaying the critical period.

A series of recent reports indicates that reduced feedforward inhibition is a common feature in several mouse models of ASD (Antoine et al. 2019; Banerjee et al. 2016). Across at least five different mouse models of ASD, feedforward inhibition was found to be much more impaired than feedforward excitation, resulting in a shifted $E / I$ balance towards excitation. Our results are consistent with this interpretation, as we find a decreased GABAergic transmission (Fig. 4). In Ehmt1-deficient cells from rodents and humans, excitatory transmission is not impaired at resting membrane potential, but summation of excitatory input is impacted by an upregulation of NMDA receptors (Frega et al. 2019, 2020). Antoine et al. (2019) argue that excitation and inhibition are matched to yield stable in vivo firing patterns. This homeostatic regulation might still prove maladaptive in some situations, resulting in widened integration time windows and an inability to compensate for extremely high or low input levels. Also 
here, Ehmtl ${ }^{+/-}$mice fit the template of impaired adaptation, as excitatory synaptic scaling-up in response to input deprivation is impaired in $\mathrm{Ehmtl}^{+/-}$mice (Benevento et al. 2016). To summarize, in $E h m t 1^{+/-}$mice we find a regionand layer-specific developmental delay of $\mathrm{PV}^{+}$neuron maturation in the three main sensory cortical areas. $\mathrm{PV}^{+}$ neurons also are functionally immature, showing a reduced inhibitory output specifically caused by a reduced release probability. As a consequence, we predict a delayed closing of the critical period in auditory and somatosensory cortex. Both the onset and the closing of the critical period require coordinated changes in gene expression in $\mathrm{PV}^{+}$ neurons (Apulei et al. 2019), which has been shown to require epigenetic remodeling such as by the DNA methylation reader MeCP2 (Krishnan et al. 2015), which interestingly was recently shown to be a direct target of Ehmtl in non-neuronal cells (Choi et al. 2018). It is therefore conceivable that a loss of Ehmtl would lead to a cellautonomous failure of epigenetic remodeling, resulting in an incomplete repression of previously expressed gene sets and thus a slowed transition into and out of the CP. This concept could be generalized to other changes in gene expression, and indeed is consistent with activity-dependent scaling of excitatory synapses in Ehmt1 ${ }^{+/-}$neurons (Benevento et al. 2016) and activity-dependent rapid methylation of early-response genes by EHMT1 and EHMT2 (Gupta-Agarwal et al. 2012). We predict that finding out how the epigenetic landscape changes in inhibitory neurons - and when those changes fail-will be an important addition to solving the secret of the dynamic brain.

Acknowledgements The authors are grateful to Jon-Ruben van Rhijn and Naoki Kogo for help with the electrophysiological procedures, to Bram Geenen, Gert-Jan Bakker, Dewi van der Geugten and Katharina Foreman for initial help with the iDISCO protocol and imaging, and to Maren Bormann, Bram Bosch and Carleen Rossing for help with setting up the iDISCO analysis pipeline. We thank Tansu Celikel (Donders Institute, Nijmegen) and Michael Valente (RIMLS, Nijmegen) for the kind gifts of PV-Cre and Tdtom-flox mice, respectively. Furthermore, we would like to thank Astrid Oudakker and Anne Mulders for help with genotyping the mice, and the animal care takers from the CDL Nijmegen for help with animal husbandry.

Author contributions $\mathrm{MN}$ and NNK conceptualized the project; MN, $\mathrm{KP}, \mathrm{RH}$, and LA executed immunostainings and analyzed data; MN executed all electrophysiology and analyzed data; LL performed iDISCO + staining, MN imaged and analyzed iDISCO-cleared brains; DS helped with Statistical analysis and co-supervised students; MN drafted paper; MN and NNK revised paper; NNK acquired funding.

Funding This work was supported by grants from the Netherlands Organization for Scientific Research, NWO-CAS grant 012.200.001 (to N.N.K). None of the funders had any influence on the conceptualization or execution of this study, or decision to publish.

\section{Compliance with ethical standards}

Conflict of interest The authors declare no competing financial interests.

Human and animal rights The experimental animal procedures were approved by the Animal Ethics Committee of the Radboud University Nijmegen (DEC) and the National Animal Experiment Committee (CCD), under CCD application number 2015-0038 (Nijmegen, the Netherlands) and conducted in accordance with all applicable Dutch and European legislation. Every effort was made to minimize animal discomfort and the number of animals used. No human participants were used in this study.

Open Access This article is licensed under a Creative Commons Attribution 4.0 International License, which permits use, sharing, adaptation, distribution and reproduction in any medium or format, as long as you give appropriate credit to the original author(s) and the source, provide a link to the Creative Commons licence, and indicate if changes were made. The images or other third party material in this article are included in the article's Creative Commons licence, unless indicated otherwise in a credit line to the material. If material is not included in the article's Creative Commons licence and your intended use is not permitted by statutory regulation or exceeds the permitted use, you will need to obtain permission directly from the copyright holder. To view a copy of this licence, visit http://creativecommons.org/licenses/by/4.0/.

\section{References}

Antoine MW, Langberg T, Schnepel P, Feldman DE (2019) Increased excitation-inhibition ratio stabilizes synapse and circuit excitability in four autism mouse models. Neuron 101:648-661.e4. https ://doi.org/10.1016/j.neuron.2018.12.026

Apulei J, Kim N, Testa D, Ribot J, Morizet D, Bernard C et al (2019) Non-cell autonomous OTX2 homeoprotein regulates visual cortex plasticity through Gadd45b/g. Cereb Cortex 29:2384-2395. https ://doi.org/10.1093/cercor/bhy108

Balemans MCM, Ansar M, Oudakker AR, van Caam APM, Bakker B, Vitters EL et al (2014) Reduced Euchromatin histone methyltransferase 1 causes developmental delay, hypotonia, and cranial abnormalities associated with increased bone gene expression in Kleefstra syndrome mice. Dev Biol 386:395-407. https://doi. org/10.1016/j.ydbio.2013.12.016

Balemans MCM, Huibers MMH, Eikelenboom NWD, Kuipers AJ, van Summeren RCJ, Pijpers MMCA et al (2010) Reduced exploration, increased anxiety, and altered social behavior: Autistic-like features of euchromatin histone methyltransferase 1 heterozygous knockout mice. Behav Brain Res 208:47-55. https://doi. org/10.1016/j.bbr.2009.11.008

Balemans MCM, Nadif kasri N, Kopanitsa MV, Afinowi NO, Ramakers G, Peters TA et al (2013) Hippocampal dysfunction in the Euchromatin histone methyltransferase 1 heterozygous knockout mouse model for Kleefstra syndrome. Hum Mol Genet 22:852866. https://doi.org/10.1093/hmg/dds490

Banerjee A, Rikhye RV, Breton-Provencher V, Tangb X, Li C, Li K et al (2016) Jointly reduced inhibition and excitation underlies circuit-wide changes in cortical processing in Rett syndrome. Proc Natl Acad Sci USA 113:E7287-E7296. https://doi.org/10.1073/ pnas. 1615330113 
Barkat TR, Polley DB, Hensch TK (2011) A critical period for auditory thalamocortical connectivity. Nat Neurosci 14:1189-1996. https ://doi.org/10.1038/nn.2882

Benevento M, Iacono G, Selten M, Ba W, Oudakker A, Frega M et al (2016) Histone methylation by the Kleefstra syndrome protein EHMT1 mediates homeostatic synaptic scaling. Neuron 91:341355. https://doi.org/10.1016/j.neuron.2016.06.003

Benevento $M$, van de Molengraft $M$, van Westen $R$, van Bokhoven $\mathrm{H}$, Kasri NN (2015) The role of chromatin repressive marks in cognition and disease: a focus on the repressive complex GLP/ G9a. Neurobiol Learn Mem 124:88-96. https://doi.org/10.1016/j. nlm.2015.06.013

Beurdeley M, Spatazza J, Lee HHC, Sugiyama S, Bernard C, Di Nardo AA et al (2012) Otx2 binding to perineuronal nets persistently regulates plasticity in the mature visual cortex. J Neurosci 32:94299437. https://doi.org/10.1523/JNEUROSCI.0394-12.2012

Bhumika S, Nakamura M, Valerio P, Solyga M, Lindén H, Barkat TR (2020) A late critical period for frequency modulated sweeps in the mouse auditory system. Cereb Cortex 30:2586-2599. https ://doi.org/10.1093/cercor/bhz262

Booker SA, Vida I (2018) Morphological diversity and connectivity of hippocampal interneurons. Cell Tissue Res 373:619-641. https://doi.org/10.1007/s00441-018-2882-2

Butt SJ, Stacey JA, Teramoto Y, Vagnoni C (2017) A role for GABAergic interneuron diversity in circuit development and plasticity of the neonatal cerebral cortex. Curr Opin Neurobiol 43:149-155. https://doi.org/10.1016/j.conb.2017.03.011

Chang CL, Trimbuch T, Chao HT, Jordan JC, Herman MA, Rosenmund C (2014) Investigation of synapse formation and function in a glutamatergic-GABAergic two-neuron microcircuit. J Neurosci 34:855-868. https://doi.org/10.1523/JNEUR OSCI.0229-13.2014

Choi MH, Palanichamy Kala M, Ow JR, Rao VK, Suriyamurthy S, Taneja R (2018) GLP inhibits heterochromatin clustering and myogenic differentiation by repressing MeCP2. J Mol Cell Biol 10:161-174. https://doi.org/10.1093/jmcb/mjx038

Devienne G, Picaud S, Cohen I, Piquet J, Tricoire L, Testa D, Di Nardo A, Rossier J, Cauli B, Lambolez B (2019). Regulation of Perineuronal Nets in the Adult Cortex by the Electrical Activity of Parvalbumin Interneurons. BioRxiv. https://www.biorxiv.org/ content/10.1101/671719v1

Du X, Serena K, Hwang W, Grech AM, Wu YWC, Schroeder A, Hill RA (2018) Prefrontal cortical parvalbumin and somatostatin expression and cell density increase during adolescence and are modified by BDNF and sex. Mol Cell Neurosci 88:177-188. https ://doi.org/10.1016/j.men.2018.02.001

Durand S, Patrizi A, Quast KB, Hachigian L, Pavlyuk R, Saxena A et al (2012) NMDA receptor regulation prevents regression of visual cortical function in the absence of Mecp2. Neuron 76:1078-1090. https://doi.org/10.1016/j.neuron.2012.12.004

Ebbers L, Runge K, Nothwang HG (2016) Differential patterns of histone methylase EHMT2 and its catalyzed histone modifications $\mathrm{H} 3 \mathrm{~K} 9 \mathrm{me} 1$ and $\mathrm{H} 3 \mathrm{~K} 9 \mathrm{me} 2$ during maturation of central auditory system. Cell Tissue Res 365:247-264. https://doi.org/10.1007/ s00441-016-2401-2

Elmqvist BYD, Quastel DMJ (1965) A quantitative study of end-plate potentials in isolated human muscle. J Physiol 178:505-529. https ://doi.org/10.1113/jphysiol.1965.sp007639

Espinosa JS, Stryker MP (2012) Development and plasticity of the primary visual cortex. Neuron 75:230-249. https://doi.org/10.1016/j. neuron.2012.06.009

Favuzzi E, Deogracias R, Marques-Smith A, Maeso P, Jezequel J, Exposito-Alonso D et al (2019) Neurodevelopment: distinct molecular programs regulate synapse specificity in cortical inhibitory circuits. Science 363:413-417. https://doi.org/10.1126/scien ce.aau8977
Favuzzi E, Marques-Smith A, Deogracias R, Winterflood CM, Sánchez-Aguilera A, Mantoan L et al (2017) Activity-dependent gating of parvalbumin interneuron function by the perineuronal net protein Brevican. Neuron 95:639-655.e10. https://doi. org/10.1016/j.neuron.2017.06.028

Frega M, Linda K, Keller JM, Gümüş-Akay G, Mossink B, van Rhijn JR et al (2019) Neuronal network dysfunction in a model for Kleefstra syndrome mediated by enhanced NMDAR signaling. Nat Commun 10:1-15. https://doi.org/10.1038/s41467-019-12947 $-3$

Frega M, Selten M, Mossink B, Keller JM, Linda K, Moerschen R et al (2020) Distinct pathogenic genes causing intellectual disability and Autism exhibit a common neuronal network hyperactivity phenotype. Cell Rep 30:173-186.e6. https://doi.org/10.1016/j. celrep.2019.12.002

Goel A, Cantu D, Guilfoyle J, Chaudhari GR, Newadkar A, Todisco B et al (2018) Impaired perceptual learning in Fragile $X$ syndrome is mediated by parvalbumin neuron dysfunction in V1 and is reversible. Nat Neurosci. https://doi.org/10.1101/217414

Gupta-Agarwal S, Franklin AV, DeRamus T, Wheelock M, Davis RL, McMahon LL et al (2012) G9a/GLP histone lysine dimethyltransferase complex activity in the hippocampus and the entorhinal cortex is required for gene activation and silencing during memory consolidation. J Neurosci 32:5440-5453. https://doi.org/10.1523/ jneurosci.0147-12.2012

Gupta-Agarwal S, Jarome TJ, Fernandez J, Lubin FD (2014) NMDA receptor- and ERK-dependent histone methylation changes in the lateral amygdala bidirectionally regulate fear memory formation. Learn Mem 21:351-362. https://doi.org/10.1101/lm.035105.114

Harlow EG, Till SM, Russell T, Wijetunge LS, Kind P, Contractor A (2010) Critical period plasticity is disrupted in the barrel cortex of FMR1 knockout mice. Neuron 65:385-398. https://doi. org/10.1016/j.neuron.2010.01.024

He LJ, Liu N, Cheng TL, Chen XJ, Li YD, Shu YS et al (2014) Conditional deletion of Mecp2 in parvalbumin-expressing GABAergic cells results in the absence of critical period plasticity. Nat Commun. https://doi.org/10.1038/ncomms6036

Hensch TK (2005) Critical period plasticity in local cortical circuits. Nat Rev Neurosci 6:877-888. https://doi.org/10.1038/nrn1787

Hensch TK, Fagiolini M (2005) Excitatory-inhibitory balance and critical period plasticity in developing visual cortex. Prog Brain Res. https://doi.org/10.1016/S0079-6123(04)47009-5

Huang ZJ, Kirkwood A, Pizzorusso T, Porciatti V, Morales B, Bear MF, Maffei L, Tonegawa S (1999) BDNF regulates the maturation of inhibition and the critical period of plasticity in mouse visual cortex. Cell 98(6):739-755. https://doi.org/10.1016/S0092 -8674(00)81509-3

Iacono G, Dubos A, Méziane H, Benevento M, Habibi E, Mandoli A et al (2018) Increased H3K9 methylation and impaired expression of Protocadherins are associated with the cognitive dysfunctions of the Kleefstra syndrome. Nucleic Acids Res 46:4950-4965. https://doi.org/10.1093/nar/gky196

Iwase S, Bérubé NG, Zhou Z, Kasri NN, Battaglioli E, Scandaglia $M$ et al (2017) epigenetic etiology of intellectual disability. J Neurosci 37:10773-10782. https://doi.org/10.1523/JNEUR OSCI.1840-17.2017

Juczewski K, von Richthofen H, Bagni C, Celikel T, Fisone G, Krieger $\mathrm{P}$ (2016) Somatosensory map expansion and altered processing of tactile inputs in a mouse model of fragile $\mathrm{X}$ syndrome. Neurobiol Dis 96:201-215. https://doi.org/10.1016/j.nbd.2016.09.007

Kalish BT, Barkat TR, Diel EE, Zhang EJ, Greenberg ME, Hensch TK (2020) SI: Single-nucleus RNA sequencing of mouse auditory cortex reveals critical period triggers and brakes. Proc Natl Acad Sci. https://doi.org/10.1073/pnas.1920433117

Kleefstra T, Kramer JM, Neveling K, Willemsen MH, Koemans TS, Vissers LELM et al (2012) Disruption of an EHMT1-associated 
chromatin-modification module causes intellectual disability. Am J Hum Genet 91:73-82. https://doi.org/10.1016/j. ajhg.2012.05.003

Kleefstra T, Smidt M, Banning MJG, Oudakker AR, Van Esch H, De Brouwer APM et al (2005) Disruption of the gene Euchromatin Histone Methyl Transferase1 (Eu-HMTase1) is associated with the 9q34 subtelomeric deletion syndrome. J Med Genet 42:299-306. https://doi.org/10.1136/jmg.2004.028464

Koemans TS, Kleefstra T, Chubak MC, Stone MH, Reijnders MRF, de Munnik S et al (2017) Functional convergence of histone methyltransferases EHMT1 and KMT2C involved in intellectual disability and autism spectrum disorder. PLoS Genet. https://doi. org/10.1371/journal.pgen.1006864

Krishnan K, Lau BYB, Ewall G, Huang ZJ, Shea SD (2017) MECP2 regulates cortical plasticity underlying a learned behaviour in adult female mice. Nat Commun 8:14077. https://doi.org/10.1038/ ncomms 14077

Krishnan K, Wang B-S, Lu J, Wang L, Maffei A, Cang J et al (2015) $\mathrm{MeCP} 2$ regulates the timing of critical period plasticity that shapes functional connectivity in primary visual cortex. Proc Natl Acad Sci 112:E4782-E4791. https://doi.org/10.1073/pnas.15064 99112

Lau BYB, Krishnan K, Huang ZJ, Shea SD (2020) Maternal experience-dependent cortical plasticity in mice is circuit- and stimulusspecific and requires MECP2. J Neurosci. https://doi.org/10.1523/ jneurosci.1964-19.2019

Lau BYB, Layo DE, Emery B, Everett M, Kumar A, Stevenson P et al (2020) Lateralized expression of cortical perineuronal nets during maternal experience is dependent on MECP2. eNeuro. https://doi. org/10.1523/eneuro.0500-19.2020

Marín O (2016) Developmental timing and critical windows for the treatment of psychiatric disorders. Nat Med 22:1229-1238. https ://doi.org/10.1038/nm.4225

Martens MB, Frega M, Classen J, Epping L, Bijvank E, Benevento M et al (2016) Euchromatin histone methyltransferase 1 regulates cortical neuronal network development. Sci Rep 6:35756. https ://doi.org/10.1038/srep35756

McRae PA, Rocco MM, Kelly G, Brumberg JC, Matthews RT (2007) Sensory deprivation alters aggrecan and perineuronal net expression in the mouse barrel cortex. J Neurosci 27:5405-5413. https ://doi.org/10.1523/JNEUROSCI.5425-06.2007

Mowery TM, Kotak VC, Sanes DH (2016) The onset of visual experience gates auditory cortex critical periods. Nat Commun 7:1-11. https://doi.org/10.1038/ncomms10416

Neher E (2015) Merits and limitations of vesicle pool models in view of heterogeneous populations of synaptic vesicles. Neuron 87:1131-1142. https://doi.org/10.1016/j.neuron.2015.08.038

Oh SW, Harris JA, Ng L, Winslow B, Cain N, Mihalas S et al (2014) A mesoscale connectome of the mouse brain. Nature 508:207-214. https://doi.org/10.1038/nature13186

Oswald AMM, Reyes AD (2011) Development of inhibitory timescales in auditory cortex. Cereb Cortex 21:1351-1361. https:// doi.org/10.1093/cercor/bhq214

Patrizi A, Awad PN, Chattopadhyaya B, Li C, Di Cristo G, Fagiolini M (2019) Accelerated hyper-maturation of parvalbumin circuits in the absence of MeCP2. Cereb Cortex. https://doi.org/10.1093/ cercor/bhz085

Petersen CCH (2007) The functional organization of the barrel cortex. Neuron 56:339-355. https://doi.org/10.1016/j.neuron.2007.09.017

Preibisch S, Saalfeld S, Tomancak P (2009) Globally optimal stitching of tiled 3D microscopic image acquisitions. Bioinformatics 25:1463-1465. https://doi.org/10.1093/bioinformatics/btp184

Prochiantz A, Di Nardo AA (2015) Homeoprotein signaling in the developing and adult nervous system. Neuron 85:911-925. https ://doi.org/10.1016/j.neuron.2015.01.019
Reh RK, Dias BG, Nelson CA, Kaufer D, Werker JF, Kolb B et al (2020) Critical period regulation across multiple timescales. Proc Natl Acad Sci. https://doi.org/10.1073/pnas.1820836117

Reinhard SM, Rais M, Afroz S, Hanania Y, Pendi K, Espinoza K et al (2019) Reduced perineuronal net expression in Fmr1 KO mice auditory cortex and amygdala is linked to impaired fearassociated memory. Neurobiol Learn Mem 164:107042. https:// doi.org/10.1016/j.nlm.2019.107042

Renier N, Wu Z, Simon DJ, Yang J, Ariel P, Tessier-Lavigne M (2014) IDISCO: a simple, rapid method to immunolabel large tissue samples for volume imaging. Cell 159:896-910. https:// doi.org/10.1016/j.cell.2014.10.010

Renier N, Adams EL, Kirst C, Wu Z, Azevedo R, Kohl J et al (2016) Mapping of brain activity by automated volume analysis of immediate early genes. Cell 165:1789-1802. https://doi.org/10.1016/j. cell.2016.05.007

van Rhijn JR, Fisher SE, Vernes SC, Nadif Kasri N (2018) Foxp2 loss of function increases striatal direct pathway inhibition via increased GABA release. Brain Struct Funct 223:4211-4226. https://doi.org/10.1007/s00429-018-1746-6

Sampathkumar C, Wu YJ, Vadhvani M, Trimbuch T, Eickholt B, Rosenmund C (2016) Loss of MeCP2 disrupts cell autonomous and autocrine BDNF signaling in mouse glutamatergic neurons. eLife 5:1-23. https://doi.org/10.7554/elife.19374

Schaefer A, Sampath SC, Intrator A, Min A, Gertler TS, Surmeier DJ et al (2009) Control of cognition and adaptive behavior by the GLP/G9a epigenetic suppressor complex. Neuron 64:678-691. https://doi.org/10.1016/j.neuron.2009.11.019

Schindelin J, Arganda-Carreras I, Frise E, Kaynig V, Longair M, Pietzsch T et al (2012) Fiji: An open-source platform for biologicalimage analysis. Nat Methods 9:676-682. https://doi.org/10.1038/ nmeth.2019

Selby L, Zhang C, Sun QQ (2007) Major defects in neocortical GABAergic inhibitory circuits in mice lacking the fragile $\mathrm{X}$ mental retardation protein. Neurosci Lett 412:227-232. https://doi. org/10.1016/j.neulet.2006.11.062

Shinkai Y, Tachibana M (2011) H3K9 methyltransferase G9a and the related molecule GLP. Genes Dev 25:781-788. https://doi. org/10.1101/gad.2027411

Sigal YM, Bae H, Bogart LJ, Hensch TK, Zhuang X (2019) Structural maturation of cortical perineuronal nets and their perforating synapses revealed by superresolution imaging. Proc Natl Acad Sci 116:7071-7076. https://doi.org/10.1073/pnas.1817222116

Slaker ML, Harkness JH, Sorg BA (2016) A standardized and automated method of perineuronal net analysis using Wisteria floribunda agglutinin staining intensity. IBRO Rep 1:54-60. https:// doi.org/10.1016/j.ibror.2016.10.001

Spatazza J, Lee HHC, DiNardo AA, Tibaldi L, Joliot A, Hensch TK et al (2013) Choroid-plexus-derived Otx2 homeoprotein constrains adult cortical plasticity. Cell Rep 3:1815-1823. https:// doi.org/10.1016/j.celrep.2013.05.014

Sugiyama S, Di Nardo AA, Aizawa S, Matsuo I, Volovitch M, Prochiantz A et al (2008) Experience-dependent transfer of Otx2 homeoprotein into the visual cortex activates postnatal plasticity. Cell 134:508-520. https://doi.org/10.1016/j.cell.2008.05.054

Tachibana M, Ueda J, Fukuda M, Takeda N, Ohta T, Iwanari H et al (2005) Histone methyltransferases G9a and GLP form heteromeric complexes and are both crucial for methylation of euchromatin at H3-K9. Genes Dev 19:815-826. https://doi.org/10.1101/ gad. 1284005

Takesian AE, Jensen FE, Lippman-Bell JJ, Sun H, Hensch TK, Wang TT (2018) Early seizures prematurely unsilence auditory synapses to disrupt thalamocortical critical period plasticity. Cell Rep 23:2533-2540. https://doi.org/10.1016/j.celrep.2018.04.108 
Testa D, Prochiantz A, Di Nardo AA (2019) Perineuronal nets in brain physiology and disease. Semin Cell Dev Biol 89:125-135. https ://doi.org/10.1016/j.semcdb.2018.09.011

Tewari BP, Chaunsali L, Campbell SL, Patel DC, Goode AE, Sontheimer H (2018) Perineuronal nets decrease membrane capacitance of peritumoral fast spiking interneurons in a model of epilepsy. Nat Commun. https://doi.org/10.1038/s41467-01807113-0

Trachtenberg JT (2015) Competition, inhibition, and critical periods of cortical plasticity. Curr Opin Neurobiol 35:44-48. https://doi. org/10.1016/j.conb.2015.06.006

Tremblay R, Lee S, Rudy B (2016) GABAergic interneurons in the Neocortex: from cellular properties to circuits. Neuron 91:260 292. https://doi.org/10.1016/j.neuron.2016.06.033

Ueno H, Fujii K, Takao K, Suemitsu S, Murakami S, Kitamura N et al (2019) Alteration of parvalbumin expression and perineuronal nets formation in the cerebral cortex of aged mice. Mol Cell Neurosci 95:31-42. https://doi.org/10.1016/j.mcn.2018.12.008

Vermeulen K, de Boer A, Janzing JGE, Koolen DA, Ockeloen CW, Willemsen MH et al (2017) Adaptive and maladaptive functioning in Kleefstra syndrome compared to other rare genetic disorders with intellectual disabilities. Am J Med Genet Part A 173:18211830. https://doi.org/10.1002/ajmg.a.38280
Wen TH, Afroz S, Reinhard SM, Palacios AR, Tapia K, Binder DK, Razak KA, Ethell IM (2018) Genetic reduction of matrix metalloproteinase-9 promotes formation of perineuronal nets around parvalbumin-expressing interneurons and normalizes auditory cortex responses in developing Fmr1 knock-out mice. Cereb Cortex 28(11):3951-3964. https://doi.org/10.1093/cercor/bhx258

De Winter F, Kwok JCF, Fawcett JW, Vo TT, Carulli D, Verhaagen J (2016) The chemorepulsive protein semaphorin 3A and perineuronal net-mediated plasticity. Neural Plast. https://doi. org/10.1155/2016/3679545

Wolfe J, Mende C, Brecht M (2011) Social facial touch in Rats. Behav Neurosci 125:900-910. https://doi.org/10.1037/a0026165

Zaitsev AV, Povysheva NV, Lewis DA, Krimer LS (2007) P/Q-type, but not N-type, calcium channels mediate GABA release from fastspiking interneurons to pyramidal cells in rat prefrontal cortex. J Neurophysiol 97:3567-3573. https://doi.org/10.1152/jn.01293 .2006

Publisher's Note Springer Nature remains neutral with regard to jurisdictional claims in published maps and institutional affiliations. 